\title{
Exact traveling wave solutions of the space-time fractional complex Ginzburg-Landau equation and the space-time fractional Phi-4 equation using reliable methods
}

\author{
Sekson Sirisubtawee ${ }^{1,2^{*}}$ (D), Sanoe Koonprasert ${ }^{1,2}$, Surattana Sungnul ${ }^{1,2}$ and Takerngsak Leekparn ${ }^{1}$
}

${ }^{*}$ Correspondence:

sekson.s@sci.kmutnb.ac.th

${ }^{1}$ Department of Mathematics,

Faculty of Applied Science, King

Mongkut's University of Technology

North Bangkok, Bangkok, Thailand

${ }^{2}$ Centre of Excellence in

Mathematics, CHE, Bangkok,

Thailand

\section{Springer}

\begin{abstract}
Ultrashort pulse propagation in optical transmission lines and phenomena in particle physics can be investigated via the cubic-quintic Ginzburg-Landau equation and the Phi-4 equation, respectively. The main objective of this paper is to construct exact traveling wave solutions of the $(2+1)$-dimensional cubic-quintic Ginzburg-Landau equation and the Phi-4 equation of space-time fractional orders in the sense of the conformable fractional derivative. The method employed to solve the Ginzburg-Landau equation and the Phi-4 equation are the modified Kudryashov method and the $\left(G^{\prime} / G, 1 / G\right)$-expansion method, respectively. Several types of exact analytical solutions are obtained including reciprocal of exponential function solutions, hyperbolic function solutions, trigonometric function solutions and rational function solutions. Graphical representations and physical explanations of some of the obtained solutions are demonstrated using a range of fractional orders. All of the solutions have been verified by substitution into their corresponding equations with the aid of a symbolic software package. These methods are simple and efficient for solving the proposed equations.
\end{abstract}

Keywords: Conformable fractional derivative; Nonlinear space-time fractional complex Ginzburg-Landau equation; Nonlinear space-time fractional Phi-4 equation; Modified Kudryashov method; $\left(G^{\prime} / G, 1 / G\right)$-expansion method

\section{Introduction}

The investigation of exact solutions of nonlinear partial differential equations (NPDEs) plays an important role in study of physical phenomena in various scientific and engineering fields, such as biology, chemical kinetics, optical fiber, plasma physics and oceanic phenomena. Obtaining analytical and exact solutions of NPDEs for the above fields is important in analyzing the behaviors of the considered problems. Examples of recent developments in finding analytical and exact solutions for NPDEs are as follows. Guo and Lin used the ansatz method (e.g., a quadratic function or a linear combination of a quadratic and an exponential function) to obtain exact solutions of the $(2+1)$-dimensional

(c) The Author(s) 2019. This article is distributed under the terms of the Creative Commons Attribution 4.0 International License (http://creativecommons.org/licenses/by/4.0/), which permits unrestricted use, distribution, and reproduction in any medium, provided you give appropriate credit to the original author(s) and the source, provide a link to the Creative Commons license, and indicate if changes were made. 
Date-Jimbo-Kashiwara-Miwa (DJKM) equation [1]. Wazwaz and Kaur obtained optical solitons and singular soliton solutions of the self-focusing nonlinear Schrödinger (NLS) equation using the variational iteration method (VIM) [2]. Naghshband et al. analytically solved the generalized quintic complex Ginzburg-Landau equation using the homotopy analysis method (HAM) [3]. Seadawy employed the reductive perturbation method to construct solitary traveling wave solutions of the two-dimensional nonlinear KadomtsevPetviashvili (KP) dynamic equation in dust-acoustic plasmas [4] and the nonlinear threedimensional modified Zakharov-Kuznetsov (mZK) equation [5]. Mahmood and Yousif found novel analytical solutions for the modified Kawahara equation using the residual power series method [6]. Seadawy applied the auxiliary equation of the direct algebraic method to construct traveling wave solutions of the higher-order nonlinear Schrödinger equation [7]. In addition, the well-known efficient methods for constructing exact solutions of NPDEs, which have been utilized in this research field, are the Exp-function method [8], the $\left(G^{\prime} / G\right)$-expansion method [9], the first integral method [10], and the function transformation method [11].

In recent years, fractional-order differential equations (FDEs) associated with fractional derivatives such as the Riemann-Liouville derivative [12], the Caputo derivative [12], the Caputo-Fabrizio derivative [13] and Jumarie's modified Riemann-Liouville derivative [14] have been applied to numerous research areas such as applied mathematics, engineering, physics and finance. This is because the behavior of many systems shows after effects or memory which can be better explained using fractional-order derivatives. Thus, FDEs are expansively used to model various complex phenomena. Many effective methods used to solve nonlinear differential equations of integer order are generalized to solve FDEs analytically and numerically such as the ADM [15], the VIM [16], the finite element method [17], and the predictor-corrector scheme or PECE (predictor, evaluate, corrector and evaluate) method [18]. In particular, the $\left(G^{\prime} / G, 1 / G\right)$-expansion method [19-22], the modified Kudryashov method [23-26], the improved extended tanh-coth method [27] are used to get exact solutions of nonlinear fractional partial differential equations (NFPDEs).

In this paper, we are interested in using the modified Kudryashov method and the $\left(G^{\prime} / G, 1 / G\right)$-expansion method to solve two partial differential equations arising in physics and engineering problems. Here a review of the modified Kudryashov method is briefly given as follows. Ege and Misirli [23] used the method to solve the space-time fractional modified Benjamin-Bona-Mahony and potential Kadomtsev-Petviashvili equations. Zayed and Alurrfi [24] applied the modified Kudryashov method to seventh-order nonlinear partial differential equations including the Sawada-Kotera-Ito equation, the KaupKupershmidt equation and the Lax equation. Hosseini et al. [25] employed the method to solve the conformable time-fractional Klein-Gordon equations with quadratic and cubic nonlinear terms. The fractional generalized reaction Duffing model equation, the fractional biological population model and the fractional diffusion reaction equation were solved via the modified Kudryashov method [26]. For the $\left(G^{\prime} / G, 1 / G\right)$-expansion method, Muhammad and Syed [19] applied the method to obtain exact solutions of the positive Gardner-KP equation. Demiray et al. [20] used the $\left(G^{\prime} / G, 1 / G\right)$-expansion method to solve the new Hamiltonian amplitude equation and the Broer-Kaup equation. Zayed and Alurrfi [21] employed this method to solve two nonlinear Schrödinger equations. The method was used to find exact solutions of the $(3+1)$-dimensional space-time fractional JimboMiwa equation by Sirisubtawee et al. [22]. 
We will apply the two methods mentioned above to construct exact solutions for the following two conformable space-time fractional order partial differential equations. The first one is the fractional $(2+1)$-dimensional cubic-quintic Ginzburg-Landau equation which will be solved using the modified Kudryashov method. The second problem is the nonlinear fractional Phi-4 equation for which the $\left(G^{\prime} / G, 1 / G\right)$-expansion method will be used. Some recent work utilizing the conformable fractional derivative in real world problems such the Burgers-KdV equation, the KdV-Zakharov-Kuznetsev equation, the unstable Schrödinger equation, and the resonant nonlinear Schrödinger equation have been studied in [28-30]. In addition, exact solutions to equations associated with the above two problems have been found using many methods. Some examples are as follows. For the $(2+1)$-dimensional nonlinear cubic-quintic Ginzburg-Landau equation, Shi et al. [31] used a novel identical rewriting of the differential equation method and the Exp-function method to construct solitary solutions and periodic solutions of the equation. Zayed and Alurrfi [21] obtained traveling wave solutions using the $\left(G^{\prime} / G, 1 / G\right)$-expansion method. The new extended auxiliary equation method was applied to construct many new types of Jacobi elliptic function solutions for the equation by Zayed and Alurrfi [32]. The new mapping method was employed for finding many other new exact solutions of the equation by Zayed and Al-Nowehy [33] and they concluded that this method and the extended auxiliary equation method are efficient techniques for finding exact solutions of NPDEs in mathematical physics. For the Phi-4 equation, Akter and Akbar [34] implemented the modified simple equation (MSE) method to find a solitary wave solution of the equation. Tariq and Akram [35] utilized the tanh method along with the fractional complex transform to solve the time fractional Phi-4 equation. Khan et al. [36] applied the $\left(G^{\prime} / G\right)$ expansion method to attain new solitary wave solutions of the space-time fractional Phi-4 equation in the sense of the modified Riemann-Liouville fractional operator. Akram [37] used the modified Kudryashov method and the $\exp (-\Phi(\xi))$ method to extract the exact solutions of the nonlinear fractional Phi-4 equation in the sense of the conformable timefractional derivative.

Here the space-time fractional $(2+1)$-dimensional cubic-quintic Ginzburg-Landau equation and nonlinear fractional Phi-4 equation in the sense of the conformable fractional derivative, for which we wish to obtain exact solutions, are as follows.

1. The space-time fractional $(2+1)$-dimensional cubic-quintic Ginzburg-Landau equation [33] is written as

$$
\begin{aligned}
& i D_{z}^{\delta} u+\frac{1}{2} D_{x}^{2 \beta} u+\frac{1}{2}\left(\beta_{1}-i\right) D_{\tau}^{2 \alpha} u+i u+\left(1-i r_{1}\right) u|u|^{2}+i r_{2} u|u|^{4}=0 \\
& \quad 0<\delta, \beta, \alpha \leq 1
\end{aligned}
$$

where $D_{s}^{\eta} u$ is the conformable fractional derivative of $u$ with respect to $s$ of order $\eta$. The equation describes the propagation of optical pulses in optic fibers in which the complex function $u=u(x, z, \tau)$ is a slowly varying envelop of the electric field, the parameters $\beta_{1}<0, r_{1}$ and $r_{2}$ are real constant, and the variables $z$ and $x$ are the propagation and transverse coordinates, respectively. The variable $\tau=t-\frac{z}{V_{0}}$ is the so-called reduced time, where $t$ is the physical time, and $V_{0}$ is the group velocity of the carrier wave. 
2. The conformable space-time fractional Phi-4 equation is expressed as [36]

$$
D_{t}^{2 \alpha} u-D_{x}^{2 \beta} u+m^{2} u+n u^{3}=0, \quad 0<\alpha, \beta \leq 1,
$$

where $D_{s}^{\eta} u$ denotes the conformable fractional derivative of $u$ with respect to $s$ of order $\eta$ and $m, n$ are real constants. The equation has played an important role in particle and nuclear physics.

The rest of this paper is organized as follows. In Sect. 2, the definition of the conformable fractional derivative and some of its important properties are given. In Sect. 3, we provide the main steps of the modified Kudryashov method and the $\left(G^{\prime} / G, 1 / G\right)$-expansion method. The applications of the two methods to the mentioned fractional-order problems are presented in Sect. 4. In Sect. 5, graphs and physical explanations of some selected exact solutions of the two equations are described. Some conclusions and discussions are given in Sect. 6.

\section{The conformable fractional derivative and its properties}

In this section, the definition of the conformable fractional derivative and its vital properties are given as follows.

Definition 1 Given a function $f:[0, \infty) \rightarrow \mathbb{R}$. Then the conformable fractional derivative of $f$ of order $\alpha$ is defined by $[26,38]$

$$
D_{t}^{\alpha} f(t)=\lim _{\varepsilon \rightarrow 0} \frac{f\left(t+\varepsilon t^{1-\alpha}\right)-f(t)}{\varepsilon}, \quad \text { for all } t>0,0<\alpha \leq 1 .
$$

If the limit in Eq. (3) exists, then we say that $f$ is $\alpha$-conformable differentiable at a point $t>0$.

Theorem 1 Let $\alpha \in(0,1]$, and $f(t), g(t)$ be $\alpha$-conformable differentiable at a point $t>0$, then

$$
\begin{aligned}
& D_{t}^{\alpha}(\lambda)=0, \quad \text { where } \lambda=\text { constant, } \\
& D_{t}^{\alpha}\left(t^{\mu}\right)=\mu t^{\mu-\alpha}, \quad \text { for all } \mu \in \mathbb{R}, \\
& D_{t}^{\alpha}(a f(t)+b g(t))=a D_{t}^{\alpha} f(t)+b D_{t}^{\alpha} g(t), \quad \text { for all } a, b \in \mathbb{R}, \\
& D_{t}^{\alpha}(f(t) g(t))=f(t) D_{t}^{\alpha} g(t)+g(t) D_{t}^{\alpha} f(t), \\
& D_{t}^{\alpha}\left(\frac{f(t)}{g(t)}\right)=\frac{g(t) D_{t}^{\alpha} f(t)-f(t) D_{t}^{\alpha} g(t)}{g(t)^{2}} .
\end{aligned}
$$

Remark 1 Conformable fractional derivatives of some important functions are as follows [38].

(1) $D_{t}^{\alpha}\left(e^{c t}\right)=c t^{1-\alpha} e^{c t}, c \in \mathbb{R}$.

(2) $D_{t}^{\alpha}(\sin b t)=b t^{1-\alpha} \cos b t, b \in \mathbb{R}$.

(3) $D_{t}^{\alpha}(\cos b t)=-b t^{1-\alpha} \sin b t, b \in \mathbb{R}$.

(4) $D_{t}^{\alpha}\left(\frac{1}{\alpha} t^{\alpha}\right)=1$.

(5) $D_{t}^{\alpha}(f(t))=t^{1-\alpha} \frac{d f(t)}{d t}$, provided that $f(t)$ is differentiable. 
Theorem 2 Let $f:(0, \infty) \rightarrow \mathbb{R}$ be a function such that $f$ is differentiable and $\alpha$ conformable differentiable. Also, let $g$ be a differentiable function defined in the range off . Then

$$
D_{t}^{\alpha}(f \circ g)(t)=t^{1-\alpha} f^{\prime}(g(t)) g^{\prime}(t)
$$

where the prime notation (') denotes the classical derivative.

\section{Methods}

Consider a nonlinear fractional evolution partial differential equation in three independent variables $t, x$, and $y$ as follows:

$$
F\left(u, D_{t}^{\alpha} u, D_{x}^{\beta} u, D_{y}^{\gamma} u, D_{t}^{2 \alpha} u, D_{t}^{\alpha} D_{x}^{\beta} u, D_{t}^{\alpha} D_{y}^{\gamma} u, \ldots\right)=0, \quad 0<\alpha, \beta, \gamma \leq 1,
$$

where $D_{t}^{\alpha} u, D_{x}^{\beta} u$, and $D_{y}^{\gamma} u$ are the conformable derivatives of a dependent variable $u$ with respect to independent variables $t, x$, and $y$. $F$ is a polynomial of the unknown function $u=u(x, y, t)$ and its various partial derivatives in which the highest-order derivatives and nonlinear terms are associated. Using the following traveling wave transformation:

$$
u(x, y, t)=u(\xi), \quad \xi=\frac{k x^{\beta}}{\beta}+\frac{l y^{\gamma}}{\gamma}+\frac{c t^{\alpha}}{\alpha}
$$

where $k, l$, and $c$ are constants to be determined later, Eq. (4) is reduced to an ODE in $u=u(\xi)$,

$$
P\left(u, u^{\prime}, u^{\prime \prime}, \ldots\right)=0 \text {, }
$$

where $P$ is a polynomial of $u(\xi)$ and its various derivatives and the prime notation (') denotes the derivative with respect to $\xi$.

\subsection{The modified Kudryashov method}

The main steps of the modified Kudryashov method are as follows [23-26].

Step 1. We assume that the exact solutions of Eq. (6) can be expressed in the following form:

$$
u(\xi)=\sum_{i=0}^{N} a_{i} Q(\xi)^{i},
$$

where the coefficients $a_{i}(i=0,1, \ldots, N-1)$ and $a_{N} \neq 0$ are constants to be determined later and the function $Q$ is of the form

$$
Q(\xi)=\frac{1}{1+d a^{\xi}}, \quad \text { where } d \text { is an arbitrary constant. }
$$

$Q(\xi)$ satisfies the following differential equation:

$$
Q^{\prime}(\xi)=\left[Q^{2}(\xi)-Q(\xi)\right] \ln a,
$$


where $a>0, a \neq 1$. If $a=e$, then the method reduces to the Kudryashov method which has been proposed by many authors [39-41].

Step 2. The value of the positive integer $N$ in Eq. (7) can be computed by balancing the highest-order nonlinear term with the highest-order derivative in $u(\xi)$ occurring in Eq. (6). If the degree of $u(\xi)$ is $\operatorname{Deg}[u(\xi)]=N$, then the degree of the other terms can be expressed as

$$
\operatorname{Deg}\left[\frac{d^{q} u(\xi)}{d \xi^{q}}\right]=N+q, \quad \operatorname{Deg}\left[(u(\xi))^{p}\left(\frac{d^{q} u(\xi)}{d \xi^{q}}\right)^{s}\right]=N p+s(N+q) .
$$

Remark 2 In some nonlinear equations, if the balance number $N$ is not a positive integer (e.g., a fraction or a negative integer), then a special transformation is required. The approaches to setting such a transformation can be found in [21, 42].

Step 3. We substitute the result of Eq. (7) into Eq. (6) with the aid of Eq. (9), then we obtain polynomials in $Q(\xi)$. Collecting all coefficients of like power (i.e., $Q(\xi)^{i}, i=0,1,2, \ldots, M$, where $M$ is some positive integer) of the resulting polynomials and setting them to zero, we obtain a system of algebraic equations which can be solved using a symbolic software package to get the unknowns $a_{i}(i=0,1, \ldots, N), k, l$, and $c$.

Step 4. We substitute the obtained unknown values from Step. 3 together with the function $Q(\xi)$ in Eq. (8) into Eq. (7) to get the exact solutions of Eq. (4) with $\xi$ in Eq. (5).

\subsection{The $\left(G^{\prime} / G, 1 / G\right)$-expansion method}

Before we give the main steps of the $\left(G^{\prime} / G, 1 / G\right)$-expansion method, it is necessary to introduce the following concept [19-22]. Consider the following second-order linear ODE:

$$
G^{\prime \prime}(\xi)+\lambda G(\xi)=\mu,
$$

where the prime notation (') denotes the derivative with respect to $\xi$ and where $\lambda, \mu$ are constants. We then set the functions $\phi$ and $\psi$ as follows:

$$
\phi(\xi)=\frac{G^{\prime}(\xi)}{G(\xi)}, \quad \psi(\xi)=\frac{1}{G(\xi)} .
$$

Equations (11) and (12) can be converted into the following system of two nonlinear ordinary differential equations:

$$
\phi^{\prime}=-\phi^{2}+\mu \psi-\lambda, \quad \psi^{\prime}=-\phi \psi .
$$

The solutions of Eq. (11) can be categorized into the following three cases.

Case 1: If $\lambda<0$, then the general solution of (11) is of the form

$$
G(\xi)=A_{1} \sinh (\xi \sqrt{-\lambda})+A_{2} \cosh (\xi \sqrt{-\lambda})+\frac{\mu}{\lambda},
$$

and we have

$$
\psi^{2}=\frac{-\lambda}{\lambda^{2} \sigma_{1}+\mu^{2}}\left(\phi^{2}-2 \mu \psi+\lambda\right)
$$

where $A_{1}$ and $A_{2}$ are arbitrary constants and $\sigma_{1}=A_{1}^{2}-A_{2}^{2}$. 
Case 2: If $\lambda>0$, then the general solution of (11) can be given as

$$
G(\xi)=A_{1} \sin (\xi \sqrt{\lambda})+A_{2} \cos (\xi \sqrt{\lambda})+\frac{\mu}{\lambda},
$$

and we have the following associated relation:

$$
\psi^{2}=\frac{\lambda}{\lambda^{2} \sigma_{2}-\mu^{2}}\left(\phi^{2}-2 \mu \psi+\lambda\right)
$$

where $A_{1}$ and $A_{2}$ are arbitrary constants and $\sigma_{2}=A_{1}^{2}+A_{2}^{2}$.

Case 3: If $\lambda=0$, then the general solution of (11) can be written as

$$
G(\xi)=\frac{\mu}{2} \xi^{2}+A_{1} \xi+A_{2},
$$

and the corresponding relation is

$$
\psi^{2}=\frac{1}{A_{1}^{2}-2 \mu A_{2}}\left(\phi^{2}-2 \mu \psi\right),
$$

where $A_{1}$ and $A_{2}$ are arbitrary constants.

The main steps of the $\left(G^{\prime} / G, 1 / G\right)$-expansion method [19-22] are as follows.

Step 1. Suppose that the solution to Eq. (6) can be expressed in terms of a polynomial of the two variables $\phi$ and $\psi$ as

$$
u(\xi)=a_{0}+\sum_{j=1}^{N} a_{j} \phi^{j}+\sum_{j=1}^{N} b_{j} \phi^{j-1} \psi,
$$

where $a_{0}, a_{j}$, and $b_{j}(j=1,2, \ldots, N)$ are constants to be determined later with $a_{N}^{2}+b_{N}^{2} \neq 0$ and where the functions $\phi=\phi(\xi)$ and $\psi=\psi(\xi)$ are implicitly related to Eq. (11) via the relations in Eq. (12).

Step 2. We find the value of the positive integer $N$ which can be computed by balancing the highest-order derivative and the nonlinear terms in Eq. (6). Suppose that the degree of $u(\xi)$ is $\operatorname{Deg}[u(\xi)]=N$, then the degree of other terms will be given by Eq. (10).

Step 3. Substituting the result from Eq. (20) into Eq. (6) with the aid of Eq. (13) and Eq. (15), the function $P$ in Eq. (6) can be converted into a polynomial in $\phi$ and $\psi$, in which the degree of $\psi$ will be one. Equating each coefficient of the resulting polynomial to zero, we obtain a system of algebraic equations, which can be solved using a symbolic computational package, for the unknowns $a_{0}, a_{j}, b_{j}(j=1,2, \ldots, N), k, l, c, \mu, \lambda(<0), A_{1}$, and $A_{2}$. Hence, the exact solutions of Eq. (4) generated by this step with the transformation in Eq. (5) are obtained in terms of hyperbolic functions.

Step 4. In the same manner as Step 3, substituting the result from Eq. (20) into Eq. (6) with the aid of Eq. (13) and Eq. (17) for $\lambda>0$, we can obtain the exact solutions of Eq. (4) by using the transformation in Eq. (5). The obtained exact solutions in this step are in terms of trigonometric functions.

Step 5. Similar to Step 3, substituting the result from Eq. (20) into Eq. (6) with the aid of Eq. (13) and Eq. (19) for $\lambda=0$, we can get the traveling wave solutions of Eq. (4) with the aid of the transformation in Eq. (5). The resulting exact solutions in this step are obtained in terms of rational functions. 


\section{Exact solutions to NFPDEs}

In this section, we will apply the modified Kudryashov method to find the exact traveling wave solutions of the space-time fractional $(2+1)$-dimensional cubic-quintic GinzburgLandau equation as given by Eq. (1) and use the $\left(G^{\prime} / G, 1 / G\right)$-expansion method to obtain exact solutions of the conformable space-time fractional Phi-4 equation as given by Eq. (2).

\subsection{Obtaining exact solutions of Eq. (1) using the modified Kudryashov method}

In order to solve Eq. (1), we assume that the exact solution has the following form:

$$
u(x, z, \tau)=w(x, \tau) e^{i\left[\frac{k z^{\delta}}{\delta}+\varphi(x, \tau)\right]},
$$

where $w(x, \tau)$ and $\varphi(x, \tau)$ are real-valued functions of $x$ and $\tau$. The parameter $k$ is a real constant and the parameter $\delta$ is as defined in Eq. (1). Substituting Eq. (21) into Eq. (1) and then separating the real and imaginary parts of the resulting equation, we obtain the following equations:

$$
\begin{aligned}
\operatorname{Re}:- & k w-\frac{w\left(D_{x}^{\beta} \varphi\right)^{2}}{2}+\frac{D_{x}^{2 \beta} w}{2}-\frac{\beta_{1} w\left(D_{\tau}^{\alpha} \varphi\right)^{2}}{2}+\frac{\beta_{1} D_{\tau}^{2 \alpha} w}{2}+\frac{w D_{\tau}^{2 \alpha} \varphi}{2} \\
& +D_{\tau}^{\alpha} w D_{\tau}^{\alpha} \varphi+w^{3}=0 \\
\operatorname{Im}:- & \frac{D_{\tau}^{2 \alpha} w}{2}+\frac{w D_{x}^{2 \beta} \varphi}{2}+D_{x}^{\beta} w D_{x}^{\beta} \varphi+\frac{\beta_{1} w D_{\tau}^{2 \alpha} \varphi}{2}+\beta_{1} D_{\tau}^{\alpha} w D_{\tau}^{\alpha} \varphi+w \\
& +\frac{w\left(D_{\tau}^{\alpha} \varphi\right)^{2}}{2}-r_{1} w^{3}+r_{2} w^{5}=0,
\end{aligned}
$$

where the notation $D_{\gamma}^{\kappa} v$ is the conformable fractional derivative of order $\kappa$ of $\nu$ with respect to $\gamma$.

Let

$$
\begin{aligned}
& w(x, \tau)=w(\xi), \quad \text { where } \xi=\frac{p_{0} x^{\beta}}{\beta}-\frac{p_{1} \tau^{\alpha}}{\alpha}, \\
& \varphi(x, \tau)=\varphi(\eta), \quad \text { where } \eta=\frac{q_{0} x^{\beta}}{\beta}-\frac{q_{1} \tau^{\alpha}}{\alpha} .
\end{aligned}
$$

The parameters $p_{0}, p_{1}, q_{0}$ and $q_{1}$ in Eq. (24) are real constants to be determined later. We substitute Eq. (24) into Eqs. (22) and (23) to obtain

$$
\begin{aligned}
& \left(-k-\frac{q_{0}^{2}\left(\varphi^{\prime}\right)^{2}}{2}-\frac{\beta_{1} q_{1}^{2}\left(\varphi^{\prime}\right)^{2}}{2}+\frac{q_{1}^{2} \varphi^{\prime \prime}}{2}\right) w+\left(\frac{p_{0}^{2}}{2}+\frac{\beta_{1} p_{1}^{2}}{2}\right) w^{\prime \prime}+p_{1} q_{1} \varphi^{\prime} w^{\prime}+w^{3}=0, \\
& \left(p_{0} q_{0}+\beta_{1} p_{1} q_{1}\right) \varphi^{\prime} w^{\prime}+\left(\frac{q_{0}^{2} \varphi^{\prime \prime}}{2}+\frac{\beta_{1} q_{1}^{2} \varphi^{\prime \prime}}{2}+\frac{q_{1}^{2}\left(\varphi^{\prime}\right)^{2}}{2}+1\right) w \\
& -\frac{p_{1}^{2} w^{\prime \prime}}{2}-r_{1} w^{3}+r_{2} w^{5}=0,
\end{aligned}
$$

where the prime notation (') of $w$ and $\varphi$ denotes the derivative of $w$ and $\varphi$ with respect to $\xi$ and $\eta$, respectively. 
Setting $q_{1}=\frac{q_{0}}{\sqrt{-\beta_{1}}}, p_{1}=\frac{p_{0}}{\sqrt{-\beta_{1}}}$, and then mathematically manipulating them, we have the following relations:

$$
\frac{\left(p_{0}^{2}+\beta_{1} p_{1}^{2}\right)}{2}=0, \quad p_{0} q_{0}+\beta_{1} p_{1} q_{1}=0, \quad \frac{\left(q_{0}^{2}+\beta_{1} q_{1}^{2}\right)}{2}=0 .
$$

Using Eq. (27), Eqs. (25) and (26) can be reduced to

$$
\begin{aligned}
& \left(\frac{1}{2} q_{1}^{2} \varphi^{\prime \prime}-k\right) w+p_{1} q_{1} \varphi^{\prime} w^{\prime}+w^{3}=0 \\
& \left(\frac{1}{2} q_{1}^{2}\left(\varphi^{\prime}\right)^{2}+1\right) w-\frac{1}{2} p_{1}^{2} w^{\prime \prime}-r_{1} w^{3}+r_{2} w^{5}=0
\end{aligned}
$$

Let $\varphi(\eta)=\eta$ where $\eta$ is given in Eq. (24), then

$$
\varphi^{\prime}=1, \quad \varphi^{\prime \prime}=0 .
$$

Using Eq. (30), Eq. (28) is reduced to

$$
w^{\prime}=\frac{k w-w^{3}}{p_{1} q_{1}}, \quad \text { and then } \quad w^{\prime \prime}=\frac{\left(k-3 w^{2}\right) w^{\prime}}{p_{1} q_{1}} .
$$

From Eq. (31), we obtain

$$
w^{\prime \prime}=\frac{\left(k-3 w^{2}\right)\left(k w-w^{3}\right)}{p_{1}^{2} q_{1}^{2}} \Rightarrow\left(\frac{k}{q_{1}}\right)^{2} w-\frac{4 k}{q_{1}^{2}} w^{3}+\frac{3}{q_{1}^{2}} w^{5}-p_{1}^{2} w^{\prime \prime}=0 .
$$

Using Eq. (30), Eq. (29) can be written as

$$
\left(q_{1}^{2}+2\right) w-2 r_{1} w^{3}+2 r_{2} w^{5}-p_{1}^{2} w^{\prime \prime}=0 .
$$

Comparing the coefficients of Eqs. (32) and (33), we get the following relations:

$$
\frac{k^{2}}{q_{1}^{2}}=q_{1}^{2}+2, \quad r_{1}=\frac{2 k}{q_{1}^{2}}, \quad 2 r_{2}=\frac{3}{q_{1}^{2}} .
$$

Simplifying Eq. (34), we obtain

$$
k=\frac{3 r_{1}}{4 r_{2}}, \quad \frac{9 r_{1}^{2}}{16 r_{2}^{2}}=q_{1}^{2}\left(q_{1}^{2}+2\right),
$$

where the parameters $r_{1}$ and $r_{2}$ must satisfy the relation $3 r_{1}^{2}=16 r_{2}+12$.

Substituting the above expressions for $k$ and $q_{1}$ into Eq. (32) (or (33)), we attain an ODE as follows:

$$
3 r_{1}^{2} w-16 r_{1} r_{2} w^{3}+16 r_{2}^{2} w^{5}-8 r_{2} p_{1}^{2} w^{\prime \prime}=0 .
$$

We multiply both sides of Eq. (36) by $w^{\prime}$ and then integrate the resulting equation with respective to $\xi$ to obtain

$$
\frac{3}{2} r_{1}^{2} w^{2}-4 r_{1} r_{2} w^{4}+\frac{8}{3} r_{2}^{2} w^{6}-4 r_{2} p_{1}^{2}\left(w^{\prime}\right)^{2}+k_{0}=0
$$


where $k_{0}$ is a constant of integration. Using Eq. (10) to balance the terms $\left(w^{\prime}\right)^{2}$ and $w^{6}$ in Eq. (37), we get $N=\frac{1}{2}$. According to Remark 2 in Sect. 3, we let $v(\xi)$ be a new function satisfying the following transformation:

$$
w(\xi)=v(\xi)^{\frac{1}{2}} .
$$

Putting Eq. (38) into Eq. (37), we get the following ODE in the variable $v$ :

$$
k_{0} v+\frac{3}{2} r_{1}^{2} v^{2}-4 r_{1} r_{2} v^{3}+\frac{8}{3} r_{2}^{2} v^{4}-r_{2} p_{1}^{2}\left(v^{\prime}\right)^{2}=0
$$

Again using Eq. (10) to balance the terms $\left(v^{\prime}\right)^{2}$ and $v^{4}$ in Eq. (39), we obtain $N=1$. Therefore, the solution form of Eq. (39) is

$$
v(\xi)=a_{0}+a_{1} Q(\xi)
$$

where $a_{0}, a_{1}(\neq 0)$ are constants to be determined later. Substituting Eq. (40) along with Eq. (9) into Eq. (39) and equating all the coefficients of like power of $Q(\xi)$ to be zero, we find the following algebraic system:

$$
\begin{aligned}
& Q(\xi)^{4}: \frac{8}{3} r_{2}^{2} a_{1}^{4}-r_{2} p_{1}^{2} a_{1}^{2}(\ln a)^{2}=0, \\
& Q(\xi)^{3}:-4 r_{1} r_{2} a_{1}^{3}+2 r_{2} p_{1}^{2} a_{1}^{2}(\ln a)^{2}+\frac{32}{3} r_{2}^{2} a_{0} a_{1}^{3}=0, \\
& Q(\xi)^{2}:-12 r_{1} r_{2} a_{0} a_{1}^{2}+\frac{3}{2} r_{1}^{2} a_{1}^{2}+16 r_{2}^{2} a_{0}^{2} a_{1}^{2}-r_{2} p_{1}^{2} a_{1}^{2}(\ln a)^{2}=0, \\
& Q(\xi)^{1}: 3 r_{1}^{2} a_{0} a_{1}-12 r_{1} r_{2} a_{0}^{2} a_{1}+k_{0} a_{1}+\frac{32}{3} r_{2}^{2} a_{0}^{3} a_{1}=0, \\
& Q(\xi)^{0}: k_{0} a_{0}-4 r_{1} r_{2} a_{0}^{3}+\frac{3}{2} r_{1}^{2} a_{0}^{2}+\frac{8}{3} r_{2}^{2} a_{0}^{4}=0 .
\end{aligned}
$$

Solving system (41) with the aid of the Maple package program, we obtain the following cases.

Case 1:

$$
a_{0}=0, \quad a_{1}=\frac{3 r_{1}}{4 r_{2}}, \quad p_{1}= \pm \frac{\sqrt{\frac{6}{r_{2}}} r_{1}}{2 \ln a}, \quad k_{0}=0,
$$

where $r_{2}>0$.

Using Eqs. (8), (21), (24), (38), (40), and (42), we have the exact solution of Eq. (1) as follows:

$$
u_{1}(x, z, t)=\left[\frac{3 r_{1}}{4 r_{2}}\left(\frac{1}{1+d a^{\xi}}\right)\right]^{\frac{1}{2}} e^{i\left(\frac{3 r_{1} z^{\delta}}{4 r_{2} \delta}+\frac{\sqrt{\frac{-3 \beta_{1}}{2 r_{2}}}}{\beta} x^{\beta}-\frac{\sqrt{\frac{3}{2 r_{2}}}}{\alpha} \tau^{\alpha}\right)},
$$

where $\xi= \pm \frac{\sqrt{\frac{-6 \beta_{1}}{r_{2}}} r_{1}}{2 \beta \ln a} x^{\beta} \mp \frac{\sqrt{\frac{6}{r_{2}}} r_{1}}{2 \alpha \ln a} \tau^{\alpha}$. 
Case 2:

$$
a_{0}=\frac{3 r_{1}}{4 r_{2}}, \quad a_{1}=-\frac{3 r_{1}}{4 r_{2}}, \quad p_{1}= \pm \frac{\sqrt{\frac{6}{r_{2}}} r_{1}}{2 \ln a}, \quad k_{0}=0,
$$

where $r_{2}>0$.

Using Eqs. (8), (21), (24), (38), (40), and (44), we get the traveling wave solution of Eq. (1) as follows:

$$
u_{2}(x, z, t)=\left[\frac{3 r_{1}}{4 r_{2}}\left(1-\frac{1}{\left(1+d a^{\xi}\right)}\right)\right]^{\frac{1}{2}} e^{i\left(\frac{3 r_{1} z^{\delta}}{4 r_{2} \delta}+\frac{\sqrt{\frac{-3 \beta_{1}}{2 r_{2}}}}{\beta} x^{\beta}-\frac{\sqrt{\frac{3}{2 r_{2}}}}{\alpha} \tau^{\alpha}\right)}
$$

where $\xi= \pm \frac{\sqrt{\frac{-6 \beta_{1}}{r_{2}}} r_{1}}{2 \beta \ln a} x^{\beta} \mp \frac{\sqrt{\frac{6}{r_{2}}} r_{1}}{2 \alpha \ln a} \tau^{\alpha}$.

\subsection{Obtaining exact solutions of Eq. (2) using the $\left(G^{\prime} / G, 1 / G\right)$-expansion method}

We apply the traveling wave transformation

$$
u(x, t)=u(\xi), \quad \xi=\frac{k x^{\beta}}{\beta}-\frac{c t^{\alpha}}{\alpha}
$$

where $k$ and $c$ are arbitrary constants. Using Theorem 2 and the above transformation, the conformable fractional-order derivatives of $u$ in Eq. (2) become

$$
D_{t}^{2 \alpha} u(x, t)=c^{2} u^{\prime \prime}, \quad D_{x}^{2 \beta} u(x, t)=k^{2} u^{\prime \prime} .
$$

Hence, Eq. (2) is reduced to the following ODE in the variable $u=u(\xi)$ :

$$
\left(c^{2}-k^{2}\right) u^{\prime \prime}+n u^{3}+m^{2} u=0,
$$

where the prime notation (') denotes the derivative with respect to $\xi$.

Applying the formulas in Eq. (10) to balance the highest-order derivative and nonlinear terms, i.e., $u^{\prime \prime}$ and $u^{3}$ in Eq. (47), we obtain $N=1$. Hence the solution form of Eq. (47) is

$$
u(\xi)=a_{0}+a_{1} \phi(\xi)+b_{1} \psi(\xi)
$$

where the constant coefficients $a_{0}, a_{1}$, and $b_{1}$ are to be determined later, subject to the inequality $a_{1}^{2}+b_{1}^{2} \neq 0$. There are three cases of the function $G(\xi)$ associated with the functions $\phi(\xi)$ and $\psi(\xi)$ of the solution in Eq. (48) depending on the sign of $\lambda$, as described in Sect. 3.

Case 1 (Hyperbolic function solutions): If $\lambda<0$, we substitute Eq. (48) into Eq. (47) and utilize Eqs. (13) and (15) so that the left-hand side of Eq. (47) becomes a polynomial in $\phi(\xi)$ and $\psi(\xi)$. Setting all of the coefficients of this resulting polynomial to be zero, we 
obtain the following system of nonlinear algebraic equations in $a_{0}, a_{1}, b_{1}, k, c, \mu$ and $\lambda$ :

$$
\begin{aligned}
& \phi^{3}: \lambda^{4} n a_{1}^{3} \sigma_{1}^{2}+2 c^{2} \lambda^{4} a_{1} \sigma_{1}^{2}-2 k^{2} \lambda^{4} a_{1} \sigma_{1}^{2}+2 \lambda^{2} \mu^{2} n a_{1}^{3} \sigma_{1}+4 c^{2} \lambda^{2} \mu^{2} a_{1} \sigma_{1}+\mu^{4} n a_{1}^{3} \\
& -4 k^{2} \lambda^{2} \mu^{2} a_{1} \sigma_{1}-3 \lambda^{3} n a_{1} b_{1}^{2} \sigma_{1}+2 c^{2} \mu^{4} a_{1}-2 k^{2} \mu^{4} a_{1}-3 \lambda \mu^{2} n a_{1} b_{1}^{2}=0, \\
& \phi^{2}: 3 \lambda^{4} n a_{0} a_{1}^{2} \sigma_{1}^{2}+6 \lambda^{2} \mu^{2} n a_{0} a_{1}^{2} \sigma_{1}+c^{2} \lambda^{3} \mu b_{1} \sigma_{1}-k^{2} \lambda^{3} \mu b_{1} \sigma_{1}-3 \lambda^{3} n a_{0} b_{1}^{2} \sigma_{1} \\
& +3 \mu^{4} n a_{0} a_{1}^{2}+c^{2} \lambda \mu^{3} b_{1}-k^{2} \lambda \mu^{3} b_{1}-2 \lambda^{2} \mu n b_{1}^{3}-3 \lambda \mu^{2} n a_{0} b_{1}^{2}=0, \\
& \phi^{2} \psi: 3 \lambda^{4} n a_{1}^{2} b_{1} \sigma_{1}^{2}+2 c^{2} \lambda^{4} b_{1} \sigma_{1}^{2}-2 k^{2} \lambda^{4} b_{1} \sigma_{1}^{2}+6 \lambda^{2} \mu^{2} n a_{1}^{2} b_{1} \sigma_{1}+4 c^{2} \lambda^{2} \mu^{2} b_{1} \sigma_{1} \\
& -4 k^{2} \lambda^{2} \mu^{2} b_{1} \sigma_{1}-\lambda^{3} n b_{1}^{3} \sigma_{1}+3 \mu^{4} n a_{1}^{2} b_{1}+2 c^{2} \mu^{4} b_{1}-2 k^{2} \mu^{4} b_{1}-\lambda \mu^{2} n b_{1}^{3}=0, \\
& \phi: 2 c^{2} \lambda^{5} a_{1} \sigma_{1}^{2}-2 k^{2} \lambda^{5} a_{1} \sigma_{1}^{2}+3 \lambda^{4} n a_{0}^{2} a_{1} \sigma_{1}^{2}+4 c^{2} \lambda^{3} \mu^{2} a_{1} \sigma_{1}-4 k^{2} \lambda^{3} \mu^{2} a_{1} \sigma_{1} \\
& +\lambda^{4} m^{2} a_{1} \sigma_{1}^{2}-3 \lambda^{4} n a_{1} b_{1}^{2} \sigma_{1}+6 \lambda^{2} \mu^{2} n a_{0}^{2} a_{1} \sigma_{1}+2 c^{2} \lambda \mu^{4} a_{1}-2 k^{2} \lambda \mu^{4} a_{1} \\
& +2 \lambda^{2} m^{2} \mu^{2} a_{1} \sigma_{1}-3 \lambda^{2} \mu^{2} n a_{1} b_{1}^{2}+3 \mu^{4} n a_{0}^{2} a_{1}+m^{2} \mu^{4} a_{1}=0, \\
& \phi \psi:-3 c^{2} \lambda^{4} \mu a_{1} \sigma_{1}^{2}+3 k^{2} \lambda^{4} \mu a_{1} \sigma_{1}^{2}+6 \lambda^{4} n a_{0} a_{1} b_{1} \sigma_{1}^{2}-6 c^{2} \lambda^{2} \mu^{3} a_{1} \sigma_{1}-3 c^{2} \mu^{5} a_{1} \\
& +6 \lambda^{3} \mu n a_{1} b_{1}^{2} \sigma_{1}+12 \lambda^{2} \mu^{2} n a_{0} a_{1} b_{1} \sigma_{1}+3 k^{2} \mu^{5} a_{1}+6 \lambda \mu^{3} n a_{1} b_{1}^{2}+6 \mu^{4} n a_{0} a_{1} b_{1} \\
& +6 k^{2} \lambda^{2} \mu^{3} a_{1} \sigma_{1}=0, \\
& \psi: c^{2} \lambda^{5} b_{1} \sigma_{1}^{2}-k^{2} \lambda^{5} b_{1} \sigma_{1}^{2}+3 \lambda^{4} n a_{0}^{2} b_{1} \sigma_{1}^{2}+\lambda^{4} m^{2} b_{1} \sigma_{1}^{2}-\lambda^{4} n b_{1}^{3} \sigma_{1}+3 \mu^{4} n a_{0}^{2} b_{1} \\
& +6 \lambda^{2} \mu^{2} n a_{0}^{2} b_{1} \sigma_{1}-c^{2} \lambda \mu^{4} b_{1}+k^{2} \lambda \mu^{4} b_{1}+2 \lambda^{2} m^{2} \mu^{2} b_{1} \sigma_{1}+3 \lambda^{2} \mu^{2} n b_{1}^{3}+m^{2} \mu^{4} b_{1} \\
& +6 \lambda \mu^{3} n a_{0} b_{1}^{2}+6 \lambda^{3} \mu n a_{0} b_{1}^{2} \sigma_{1}=0, \\
& \phi^{0}: \lambda^{4} n a_{0}^{3} \sigma_{1}^{2}+c^{2} \lambda^{4} \mu b_{1} \sigma_{1}-k^{2} \lambda^{4} \mu b_{1} \sigma_{1}+\lambda^{4} m^{2} a_{0} \sigma_{1}^{2}-3 \lambda^{4} n a_{0} b_{1}^{2} \sigma_{1}+m^{2} \mu^{4} a_{0} \\
& +c^{2} \lambda^{2} \mu^{3} b_{1}-k^{2} \lambda^{2} \mu^{3} b_{1}-2 \lambda^{3} \mu n b_{1}^{3}+2 \lambda^{2} m^{2} \mu^{2} a_{0} \sigma_{1}-3 \lambda^{2} \mu^{2} n a_{0} b_{1}^{2}+\mu^{4} n a_{0}^{3} \\
& +2 \lambda^{2} \mu^{2} n a_{0}^{3} \sigma_{1}=0 \text {. }
\end{aligned}
$$

Solving the above algebraic system using the Maple package program, we get the following results.

\section{Result 1}

$$
a_{0}=0, \quad a_{1}= \pm \frac{\sqrt{-2 n\left(c^{2}-k^{2}\right)}}{n}, \quad b_{1}=0, \quad \lambda=-\frac{m^{2}}{2\left(c^{2}-k^{2}\right)}, \quad \mu=0
$$

where $c^{2}>k^{2}, n<0$ and $m$ is an arbitrary constant.

From Eqs. (14), (48), and (50), we obtain the exact solution of Eq. (2) as follows:

$$
\begin{aligned}
u_{1}(x, t)= & \pm \frac{\sqrt{-2 n\left(c^{2}-k^{2}\right)}}{n} \\
& \times\left[\sqrt{\frac{m^{2}}{2\left(c^{2}-k^{2}\right)}}\left(\frac{A_{1} \cosh \left(\xi \sqrt{\frac{m^{2}}{2\left(c^{2}-k^{2}\right)}}\right)+A_{2} \sinh \left(\xi \sqrt{\frac{m^{2}}{2\left(c^{2}-k^{2}\right)}}\right)}{A_{1} \sinh \left(\xi \sqrt{\frac{m^{2}}{2\left(c^{2}-k^{2}\right)}}\right)+A_{2} \cosh \left(\xi \sqrt{\frac{m^{2}}{2\left(c^{2}-k^{2}\right)}}\right)}\right),\right.
\end{aligned}
$$

where $\xi$ is defined in Eq. (46). 
Result 2

$$
a_{0}=0, \quad a_{1}=0, \quad b_{1}= \pm \frac{\sqrt{2 n \sigma_{1}} m}{n}, \quad \lambda=\frac{m^{2}}{\left(c^{2}-k^{2}\right)}, \quad \mu=0,
$$

where $c^{2}<k^{2}, n \sigma_{1}>0$ and $m$ is an arbitrary constant.

From Eqs. (14), (48), and (52), we have the exact solution of Eq. (2) as follows:

$$
u_{2}(x, t)=\frac{ \pm \sqrt{2 n \sigma_{1}} m}{n\left(A_{1} \sinh \left(\xi \sqrt{-\frac{m^{2}}{\left(c^{2}-k^{2}\right)}}\right)+A_{2} \cosh \left(\xi \sqrt{-\frac{m^{2}}{\left(c^{2}-k^{2}\right)}}\right)\right)}
$$

where $\xi$ is defined in Eq. (46) and $\sigma_{1}=A_{1}^{2}-A_{2}^{2} \neq 0$.

\section{Result 3}

$$
\begin{aligned}
& a_{0}=0, \quad a_{1}= \pm \frac{\sqrt{-2 n\left(c^{2}-k^{2}\right)}}{2 n}, \quad b_{1}= \pm \frac{\sqrt{-n \sigma_{1}} m}{n} \\
& \lambda=-\frac{2 m^{2}}{\left(c^{2}-k^{2}\right)}, \quad \mu=0,
\end{aligned}
$$

where $c^{2}>k^{2}, n<0, \sigma_{1}>0$ and $m$ is an arbitrary constant.

From Eqs. (14), (48), and (54), we deduce the traveling wave solution of Eq. (2) as follows:

$$
\begin{aligned}
& u_{3}(x, t)= \pm \frac{\sqrt{-2 n\left(c^{2}-k^{2}\right)}}{2 n} \\
& \times\left[\sqrt{\frac{2 m^{2}}{\left(c^{2}-k^{2}\right)}}\left(\frac{A_{1} \cosh \left(\xi \sqrt{\frac{2 m^{2}}{\left(c^{2}-k^{2}\right)}}\right)+A_{2} \sinh \left(\xi \sqrt{\frac{2 m^{2}}{\left(c^{2}-k^{2}\right)}}\right)}{A_{1} \sinh \left(\xi \sqrt{\frac{2 m^{2}}{\left(c^{2}-k^{2}\right)}}\right)+A_{2} \cosh \left(\xi \sqrt{\frac{2 m^{2}}{\left(c^{2}-k^{2}\right)}}\right)}\right)\right] \\
& \pm \frac{\sqrt{-n \sigma_{1}} m}{n\left(A_{1} \sinh \left(\xi \sqrt{\frac{2 m^{2}}{\left(c^{2}-k^{2}\right)}}\right)+A_{2} \cosh \left(\xi \sqrt{\frac{2 m^{2}}{\left(c^{2}-k^{2}\right)}}\right)\right)}
\end{aligned}
$$

where $\xi$ is defined in Eq. (46) and $\sigma_{1}=A_{1}^{2}-A_{2}^{2}$.

\section{Result 4}

$$
\begin{aligned}
& a_{0}=0, \quad a_{1}= \pm \frac{\sqrt{-2 n\left(c^{2}-k^{2}\right)}}{2 n}, \\
& b_{1}= \pm \frac{\sqrt{-n\left(c^{4} \mu^{2}-2 c^{2} k^{2} \mu^{2}+k^{4} \mu^{2}+4 m^{4} \sigma_{1}\right)}}{2 n m}, \quad \lambda=-\frac{2 m^{2}}{\left(c^{2}-k^{2}\right)}
\end{aligned}
$$

where $c^{2} \geq 2 k^{2}, n<0, m \neq 0, \sigma_{1} \geq 0$ and $\mu$ is an arbitrary constant.

From Eqs. (14), (48), and (56), we obtain the exact solution of Eq. (2) as follows:

$$
\begin{aligned}
u_{4}(x, t)= & \pm \frac{\sqrt{-2 n\left(c^{2}-k^{2}\right)}}{2 n} \\
& \times\left[\sqrt{\frac{2 m^{2}}{\left(c^{2}-k^{2}\right)}}\left(\frac{A_{1} \cosh \left(\xi \sqrt{\frac{2 m^{2}}{\left(c^{2}-k^{2}\right)}}\right)+A_{2} \sinh \left(\xi \sqrt{\frac{2 m^{2}}{\left(c^{2}-k^{2}\right)}}\right)}{A_{1} \sinh \left(\xi \sqrt{\frac{2 m^{2}}{\left(c^{2}-k^{2}\right)}}\right)+A_{2} \cosh \left(\xi \sqrt{\frac{2 m^{2}}{\left(c^{2}-k^{2}\right)}}\right)}\right)\right]
\end{aligned}
$$




$$
\pm \frac{\sqrt{-n\left(c^{4} \mu^{2}-2 c^{2} k^{2} \mu^{2}+k^{4} \mu^{2}+4 m^{4} \sigma_{1}\right)}}{2 n m\left(A_{1} \sinh \left(\xi \sqrt{\frac{2 m^{2}}{\left(c^{2}-k^{2}\right)}}\right)+A_{2} \cosh \left(\xi \sqrt{\frac{2 m^{2}}{\left(c^{2}-k^{2}\right)}}\right)\right)}
$$

where $\xi$ is defined in Eq. (46) and $\sigma_{1}=A_{1}^{2}-A_{2}^{2}$.

Case 2 (Trigonometric function solutions): If $\lambda>0$, we substitute Eq. (48) into Eq. (47) and utilize Eqs. (13) and (17) so that the left-hand side of Eq. (47) becomes a polynomial in $\phi(\xi)$ and $\psi(\xi)$. Setting all of the coefficients of the resulting polynomial to be zero, we get the system of nonlinear algebraic equations in $a_{0}, a_{1}, b_{1}, k, c, \mu$ and $\lambda$ as follows:

$$
\begin{aligned}
& \phi^{3}: \lambda^{4} n a_{1}^{3} \sigma_{2}^{2}+2 c^{2} \lambda^{4} a_{1} \sigma_{2}^{2}-2 k^{2} \lambda^{4} a_{1} \sigma_{2}^{2}-2 \lambda^{2} \mu^{2} n a_{1}^{3} \sigma_{2}-4 c^{2} \lambda^{2} \mu^{2} a_{1} \sigma_{2}+\mu^{4} n a_{1}^{3} \\
& +3 \lambda^{3} n a_{1} b_{1}^{2} \sigma_{2}+2 c^{2} \mu^{4} a_{1}-2 k^{2} \mu^{4} a_{1}-3 \lambda \mu^{2} n a_{1} b_{1}^{2}+4 k^{2} \lambda^{2} \mu^{2} a_{1} \sigma_{2}=0, \\
& \phi^{2}: 3 \lambda^{4} n a_{0} a_{1}^{2} \sigma_{2}^{2}-6 \lambda^{2} \mu^{2} n a_{0} a_{1}^{2} \sigma_{2}-c^{2} \lambda^{3} \mu b_{1} \sigma_{2}+k^{2} \lambda^{3} \mu b_{1} \sigma_{2}+3 \lambda^{3} n a_{0} b_{1}^{2} \sigma_{2} \\
& +3 \mu^{4} n a_{0} a_{1}^{2}+c^{2} \lambda \mu^{3} b_{1}-k^{2} \lambda \mu^{3} b_{1}-2 \lambda^{2} \mu n b_{1}^{3}-3 \lambda \mu^{2} n a_{0} b_{1}^{2}=0, \\
& \phi^{2} \psi: 3 \lambda^{4} n a_{1}^{2} b_{1} \sigma_{2}^{2}+2 c^{2} \lambda^{4} b_{1} \sigma_{2}^{2}-2 k^{2} \lambda^{4} b_{1} \sigma_{2}^{2}-6 \lambda^{2} \mu^{2} n a_{1}^{2} b_{1} \sigma_{2}-4 c^{2} \lambda^{2} \mu^{2} b_{1} \sigma_{2} \\
& +\lambda^{3} n b_{1}^{3} \sigma_{2}+3 \mu^{4} n a_{1}^{2} b_{1}+2 c^{2} \mu^{4} b_{1}-2 k^{2} \mu^{4} b_{1}-\lambda \mu^{2} n b_{1}^{3}+4 k^{2} \lambda^{2} \mu^{2} b_{1} \sigma_{2}=0, \\
& \phi: 2 c^{2} \lambda^{5} a_{1} \sigma_{2}^{2}-2 k^{2} \lambda^{5} a_{1} \sigma_{2}^{2}+3 \lambda^{4} n a_{0}^{2} a_{1} \sigma_{2}^{2}-4 c^{2} \lambda^{3} \mu^{2} a_{1} \sigma_{2}+4 k^{2} \lambda^{3} \mu^{2} a_{1} \sigma_{2} \\
& +3 \lambda^{4} n a_{1} b_{1}^{2} \sigma_{2}-6 \lambda^{2} \mu^{2} n a_{0}^{2} a_{1} \sigma_{2}+2 c^{2} \lambda \mu^{4} a_{1}-2 k^{2} \lambda \mu^{4} a_{1}-2 \lambda^{2} m^{2} \mu^{2} a_{1} \sigma_{2} \\
& -3 \lambda^{2} \mu^{2} n a_{1} b_{1}^{2}+3 \mu^{4} n a_{0}^{2} a_{1}+m^{2} \mu^{4} a_{1}+\lambda^{4} m^{2} a_{1} \sigma_{2}^{2}=0 \\
& \phi \psi:-3 c^{2} \lambda^{4} \mu a_{1} \sigma_{2}^{2}+3 k^{2} \lambda^{4} \mu a_{1} \sigma_{2}^{2}+6 \lambda^{4} n a_{0} a_{1} b_{1} \sigma_{2}^{2}+6 c^{2} \lambda^{2} \mu^{3} a_{1} \sigma_{2}-6 k^{2} \lambda^{2} \mu^{3} a_{1} \sigma_{2} \\
& -6 \lambda^{3} \mu n a_{1} b_{1}^{2} \sigma_{2}-12 \lambda^{2} \mu^{2} n a_{0} a_{1} b_{1} \sigma_{2}+3 k^{2} \mu^{5} a_{1}+6 \lambda \mu^{3} n a_{1} b_{1}^{2}+6 \mu^{4} n a_{0} a_{1} b_{1} \\
& -3 c^{2} \mu^{5} a_{1}=0 \\
& \psi: c^{2} \lambda^{5} b_{1} \sigma_{2}^{2}-k^{2} \lambda^{5} b_{1} \sigma_{2}^{2}+3 \lambda^{4} n a_{0}^{2} b_{1} \sigma_{2}^{2}+\lambda^{4} m^{2} b_{1} \sigma_{2}^{2}+\lambda^{4} n b_{1}^{3} \sigma_{2}-6 \lambda^{3} \mu n a_{0} b_{1}^{2} \sigma_{2} \\
& -6 \lambda^{2} \mu^{2} n a_{0}^{2} b_{1} \sigma_{2}-c^{2} \lambda \mu^{4} b_{1}+k^{2} \lambda \mu^{4} b_{1}-2 \lambda^{2} m^{2} \mu^{2} b_{1} \sigma_{2}+3 \lambda^{2} \mu^{2} n b_{1}^{3} \\
& +6 \lambda \mu^{3} n a_{0} b_{1}^{2}+3 \mu^{4} n a_{0}^{2} b_{1}+m^{2} \mu^{4} b_{1}=0, \\
& \phi^{0}: \lambda^{4} n a_{0}^{3} \sigma_{2}^{2}-c^{2} \lambda^{4} \mu b_{1} \sigma_{2}+k^{2} \lambda^{4} \mu b_{1} \sigma_{2}+\lambda^{4} m^{2} a_{0} \sigma_{2}^{2}+3 \lambda^{4} n a_{0} b_{1}^{2} \sigma_{2}-2 \lambda^{2} \mu^{2} n a_{0}^{3} \sigma_{2} \\
& +c^{2} \lambda^{2} \mu^{3} b_{1}-k^{2} \lambda^{2} \mu^{3} b_{1}-2 \lambda^{3} \mu n b_{1}^{3}-2 \lambda^{2} m^{2} \mu^{2} a_{0} \sigma_{2}-3 \lambda^{2} \mu^{2} n a_{0} b_{1}^{2}+\mu^{4} n a_{0}^{3} \\
& +m^{2} \mu^{4} a_{0}=0 \text {. }
\end{aligned}
$$

By solving the above algebraic system using the Maple package program, we obtain the following results.

\section{Result 1}

$$
a_{0}=0, \quad a_{1}= \pm \frac{\sqrt{-2 n\left(c^{2}-k^{2}\right)}}{n}, \quad b_{1}=0, \quad \lambda=-\frac{m^{2}}{2\left(c^{2}-k^{2}\right)}, \quad \mu=0
$$

where $c^{2}<k^{2}, n>0$ and $m$ is an arbitrary constant. 
From Eqs. (16), (48), and (59), we obtain the traveling wave solution of Eq. (2) as follows:

$$
\begin{aligned}
u_{1}(x, t)= & \pm \frac{\sqrt{-2 n\left(c^{2}-k^{2}\right)}}{n} \\
& \times\left[\sqrt{-\frac{m^{2}}{2\left(c^{2}-k^{2}\right)}}\left(\frac{A_{1} \cos \left(\xi \sqrt{-\frac{m^{2}}{2\left(c^{2}-k^{2}\right)}}\right)-A_{2} \sin \left(\xi \sqrt{-\frac{m^{2}}{2\left(c^{2}-k^{2}\right)}}\right)}{A_{1} \sin \left(\xi \sqrt{-\frac{m^{2}}{2\left(c^{2}-k^{2}\right)}}\right)+A_{2} \cos \left(\xi \sqrt{-\frac{m^{2}}{2\left(c^{2}-k^{2}\right)}}\right)}\right)\right]
\end{aligned}
$$

where $\xi$ is defined in Eq. (46).

\section{Result 2}

$$
a_{0}=0, \quad a_{1}=0, \quad b_{1}= \pm \frac{\sqrt{-2 n \sigma_{2}} m}{n}, \quad \lambda=\frac{m^{2}}{\left(c^{2}-k^{2}\right)}, \quad \mu=0
$$

where $c^{2}>k^{2}, n<0$ and $m$ is an arbitrary constant.

From Eqs. (16), (48), and (61), we get the exact solutions of Eq. (2) as follows:

$$
u_{2}(x, t)=\frac{ \pm \sqrt{-2 n \sigma_{2}} m}{n\left(A_{1} \sin \left(\xi \sqrt{\frac{m^{2}}{\left(c^{2}-k^{2}\right)}}\right)+A_{2} \cos \left(\xi \sqrt{\frac{m^{2}}{\left(c^{2}-k^{2}\right)}}\right)\right.},
$$

where $\xi$ is defined in Eq. (46) and $\sigma_{2}=A_{1}^{2}+A_{2}^{2}$.

\section{Result 3}

$$
\begin{aligned}
& a_{0}=0, \quad a_{1}= \pm \frac{\sqrt{-2 n\left(c^{2}-k^{2}\right)}}{2 n}, \quad b_{1}= \pm \frac{\sqrt{n \sigma_{2}} m}{n} \\
& \lambda=-\frac{2 m^{2}}{\left(c^{2}-k^{2}\right)}, \quad \mu=0,
\end{aligned}
$$

where $c^{2}<k^{2}, n>0$ and $m$ is an arbitrary constant.

From Eqs. (16), (48), and (63), we have the exact solution of Eq. (2) as follows:

$$
\begin{aligned}
& u_{3}(x, t)= \pm \frac{\sqrt{-2 n\left(c^{2}-k^{2}\right)}}{2 n} \\
& \times\left[\sqrt{-\frac{2 m^{2}}{\left(c^{2}-k^{2}\right)}}\left(\frac{A_{1} \cos \left(\xi \sqrt{-\frac{2 m^{2}}{\left(c^{2}-k^{2}\right)}}\right)-A_{2} \sin \left(\xi \sqrt{-\frac{2 m^{2}}{\left(c^{2}-k^{2}\right)}}\right)}{A_{1} \sin \left(\xi \sqrt{-\frac{2 m^{2}}{\left(c^{2}-k^{2}\right)}}\right)+A_{2} \cos \left(\xi \sqrt{-\frac{2 m^{2}}{\left(c^{2}-k^{2}\right)}}\right)}\right)\right] \\
& \pm \frac{\sqrt{n \sigma_{2}} m}{n\left(A_{1} \sin \left(\xi \sqrt{-\frac{2 m^{2}}{\left(c^{2}-k^{2}\right)}}\right)+A_{2} \cos \left(\xi \sqrt{-\frac{2 m^{2}}{\left(c^{2}-k^{2}\right)}}\right)\right.} \text {, }
\end{aligned}
$$

where $\xi$ is defined in Eq. (46) and $\sigma_{2}=A_{1}^{2}+A_{2}^{2}$. 


\section{Result 4}

$$
\begin{aligned}
& a_{0}=0, \quad a_{1}= \pm \frac{\sqrt{-2 n\left(c^{2}-k^{2}\right)}}{2 n}, \\
& b_{1}= \pm \frac{\sqrt{-n\left(c^{4} \mu^{2}-2 c^{2} k^{2} \mu^{2}+k^{4} \mu^{2}-4 m^{4} \sigma_{2}\right)}}{2 n m}, \quad \lambda=-\frac{2 m^{2}}{\left(c^{2}-k^{2}\right)},
\end{aligned}
$$

where $c^{2}<k^{2}, n>0, m \neq 0$ and $\mu$ is an arbitrary constant.

From Eqs. (16), (48), and (65), we obtain the exact solution of Eq. (2) as follows:

$$
\begin{aligned}
u_{4}(x, t)= & \pm \frac{\sqrt{-2 n\left(c^{2}-k^{2}\right)}}{2 n} \\
& \times\left[\sqrt{-\frac{2 m^{2}}{\left(c^{2}-k^{2}\right)}}\left(\frac{A_{1} \cos \left(\xi \sqrt{-\frac{2 m^{2}}{\left(c^{2}-k^{2}\right)}}\right)-A_{2} \sin \left(\xi \sqrt{-\frac{2 m^{2}}{\left(c^{2}-k^{2}\right)}}\right)}{A_{1} \sin \left(\xi \sqrt{-\frac{2 m^{2}}{\left(c^{2}-k^{2}\right)}}\right)+A_{2} \cos \left(\xi \sqrt{-\frac{2 m^{2}}{\left(c^{2}-k^{2}\right)}}\right)}\right)\right. \\
& \pm \frac{\sqrt{-n\left(c^{4} \mu^{2}-2 c^{2} k^{2} \mu^{2}+k^{4} \mu^{2}-4 m^{4} \sigma_{2}\right)}}{2 n m\left(A_{1} \sin \left(\xi \sqrt{-\frac{2 m^{2}}{\left(c^{2}-k^{2}\right)}}\right)+A_{2} \cos \left(\xi \sqrt{\left.-\frac{2 m^{2}}{\left(c^{2}-k^{2}\right)}\right)}\right.\right.}
\end{aligned}
$$

where $\xi$ is defined in Eq. (46) and $\sigma_{2}=A_{1}^{2}+A_{2}^{2}$.

Case 3 (Rational function solutions): If $\lambda=0$, we substitute Eq. (48) into Eq. (47) and utilize Eqs. (13) and (19) so that left-hand side of Eq. (47) turns out to be a polynomial in $\phi(\xi)$ and $\psi(\xi)$. Setting all of the coefficients of this resulting polynomial to zero, we obtain the system of nonlinear algebraic equations in $a_{0}, a_{1}, b_{1}, k, c$ and $\mu$ as follows:

$$
\begin{aligned}
& \phi^{3}: 4 \mu^{2} n A_{2}^{2} a_{1}^{3}-4 \mu n A_{1}^{2} A_{2} a_{1}^{3}+n A_{1}^{4} a_{1}^{3}+8 c^{2} \mu^{2} A_{2}^{2} a_{1}-8 c^{2} \mu A_{1}^{2} A_{2} a_{1}+2 c^{2} A_{1}^{4} a_{1} \\
& -8 k^{2} \mu^{2} A_{2}^{2} a_{1}+8 k^{2} \mu A_{1}^{2} A_{2} a_{1}-2 k^{2} A_{1}^{4} a_{1}-6 \mu n A_{2} a_{1} b_{1}^{2}+3 n A_{1}^{2} a_{1} b_{1}^{2}=0, \\
& \phi^{2}: 12 \mu^{2} n A_{2}^{2} a_{0} a_{1}^{2}-12 \mu n A_{1}^{2} A_{2} a_{0} a_{1}^{2}+3 n A_{1}^{4} a_{0} a_{1}^{2}+2 c^{2} \mu^{2} A_{2} b_{1}-c^{2} \mu A_{1}^{2} b_{1} \\
& -2 k^{2} \mu^{2} A_{2} b_{1}+k^{2} \mu A_{1}^{2} b_{1}-6 \mu n A_{2} a_{0} b_{1}^{2}+3 n A_{1}^{2} a_{0} b_{1}^{2}-2 \mu n b_{1}^{3}=0, \\
& \phi^{2} \psi: 12 \mu^{2} n A_{2}^{2} a_{1}^{2} b_{1}-12 \mu n A_{1}^{2} A_{2} a_{1}^{2} b_{1}+3 n A_{1}^{4} a_{1}^{2} b_{1}+8 c^{2} \mu^{2} A_{2}^{2} b_{1}-8 c^{2} \mu A_{1}^{2} A_{2} b_{1} \\
& +2 c^{2} A_{1}^{4} b_{1}-8 k^{2} \mu^{2} A_{2}^{2} b_{1}+8 k^{2} \mu A_{1}^{2} A_{2} b_{1}-2 k^{2} A_{1}^{4} b_{1}-2 \mu n A_{2} b_{1}^{3}+n A_{1}^{2} b_{1}^{3}=0, \\
& \phi: 12 \mu^{2} n A_{2}^{2} a_{0}^{2} a_{1}-12 \mu n A_{1}^{2} A_{2} a_{0}^{2} a_{1}+3 n A_{1}^{4} a_{0}^{2} a_{1}+4 m^{2} \mu^{2} A_{2}^{2} a_{1}-4 m^{2} \mu A_{1}^{2} A_{2} a_{1} \\
& +m^{2} A_{1}^{4} a_{1}=0 \\
& \phi \psi:-12 c^{2} \mu^{3} A_{2}^{2} a_{1}+12 c^{2} \mu^{2} A_{1}^{2} A_{2} a_{1}-3 c^{2} \mu A_{1}^{4} a_{1}+12 k^{2} \mu^{3} A_{2}^{2} a_{1}-12 k^{2} \mu^{2} A_{1}^{2} A_{2} a_{1} \\
& +3 k^{2} \mu A_{1}^{4} a_{1}+24 \mu^{2} n A_{2}^{2} a_{0} a_{1} b_{1}-24 \mu n A_{1}^{2} A_{2} a_{0} a_{1} b_{1}+6 n A_{1}^{4} a_{0} a_{1} b_{1} \\
& +12 \mu^{2} n A_{2} a_{1} b_{1}^{2}-6 \mu n A_{1}^{2} a_{1} b_{1}^{2}=0, \\
& \psi: 12 \mu^{2} n A_{2}^{2} a_{0}^{2} b_{1}-12 \mu n A_{1}^{2} A_{2} a_{0}^{2} b_{1}+3 n A_{1}^{4} a_{0}^{2} b_{1}-4 c^{2} \mu^{3} A_{2} b_{1}+2 c^{2} \mu^{2} A_{1}^{2} b_{1} \\
& +4 k^{2} \mu^{3} A_{2} b_{1}-2 k^{2} \mu^{2} A_{1}^{2} b_{1}+4 m^{2} \mu^{2} A_{2}^{2} b_{1}-4 m^{2} \mu A_{1}^{2} A_{2} b_{1}+m^{2} A_{1}^{4} b_{1} \\
& +12 \mu^{2} n A_{2} a_{0} b_{1}^{2}-6 \mu n A_{1}^{2} a_{0} b_{1}^{2}+4 \mu^{2} n b_{1}^{3}=0, \\
& \phi^{0}: 4 \mu^{2} n A_{2}^{2} a_{0}^{3}-4 \mu n A_{1}^{2} A_{2} a_{0}^{3}+n A_{1}^{4} a_{0}^{3}+4 m^{2} \mu^{2} A_{2}^{2} a_{0}-4 m^{2} \mu A_{1}^{2} A_{2} a_{0}+m^{2} A_{1}^{4} a_{0}=0 \text {. }
\end{aligned}
$$


Using the Maple package program to solve the above algebraic system, we obtain the following results.

\section{Result 1}

$$
a_{0}=0, \quad a_{1}=0, \quad b_{1}= \pm \frac{\sqrt{-2 n\left(c^{2}-k^{2}\right)} A_{1}}{n}, \quad \mu=0, \quad m=0,
$$

where $n\left(c^{2}-k^{2}\right)<0$ and $A_{1} \neq 0$.

From Eqs. (18), (48), and (68), we get the exact solutions of Eq. (2) as follows:

$$
u_{1}(x, t)= \pm \frac{\sqrt{-2 n\left(c^{2}-k^{2}\right)} A_{1}}{n\left(\xi A_{1}+A_{2}\right)}
$$

where $\xi$ is defined in Eq. (46) and $A_{2}$ is an arbitrary constant.

\section{Result 2}

$$
\begin{aligned}
& a_{0}=0, \quad a_{1}= \pm \frac{\sqrt{-2 n\left(c^{2}-k^{2}\right)}}{2 n}, \\
& b_{1}= \pm \frac{\sqrt{2 n\left(2 c^{2} \mu A_{2}-c^{2} A_{1}^{2}-2 k^{2} \mu A_{2}+k^{2} A_{1}^{2}\right)}}{2 n}, \quad m=0,
\end{aligned}
$$

where $n\left(c^{2}-k^{2}\right)<0, n\left(2 c^{2} \mu A_{2}-c^{2} A_{1}^{2}-2 k^{2} \mu A_{2}+k^{2} A_{1}^{2}\right)>0$ and $\mu$ is an arbitrary constant.

From Eqs. (18), (48), and (70), we attain the traveling wave solution of Eq. (2) as follows:

$$
u_{2}(x, t)=\frac{\sqrt{-2 n\left(c^{2}-k^{2}\right)}\left(\mu \xi+A_{1}\right)}{2 n\left(\frac{\mu}{2} \xi^{2}+A_{1} \xi+A_{2}\right)} \pm \frac{\sqrt{2 n\left(2 c^{2} \mu A_{2}-c^{2} A_{1}^{2}-2 k^{2} \mu A_{2}+k^{2} A_{1}^{2}\right)}}{2 n\left(\frac{\mu}{2} \xi^{2}+A_{1} \xi+A_{2}\right)},
$$

where $\xi$ is defined in Eq. (46).

\section{Graphical representations and their physical explanations for selected solutions}

In this section, we will provide some graphical representations of the exact solutions of the space-time fractional $(2+1)$-dimensional cubic-quintic Ginzburg-Landau equation as in Eq. (1) and the space-time fractional Phi-4 equation as in Eq. (2). Additionally, we will also discuss their physical explanations. The selected exact explicit solutions of Eq. (1) are plotted on $-10 \leq x \leq 10$ and $-5 \leq \tau \leq 5$ while the variable $z$ is temporarily ignored, i.e., $z=0$. The selected solutions of Eq. (2) are portrayed on $-10 \leq x \leq 10$ and $-5 \leq t \leq 5$. The fractional orders $\alpha, \beta$ of both equations are varied among $1,0.9$ and 0.8 . The graphical results of the selected solutions of each problem are described below.

The fixed values $r_{1}=2 \sqrt{5}, r_{2}=3, \beta_{1}=-1, d=1, a=2$ and the variation of $\alpha, \beta \in$ $\{1,0.9,0.8\}$ are used to plot the real part of the exact solution $u_{2}(x, z, \tau)$ in Eq. (45) of Eq. (1), which is constructed using the modified Kudryashov method. The graphs of its real part are shown in Fig. 1. They represent the oscillatory wave solutions.

Graphical representations are portrayed below using the selected exact solutions of Eq. (2) constructed from the $\left(G^{\prime} / G, 1 / G\right)$-expansion method. Using the fixed values $m=2$, $n=-3, k=1, c=9, A_{1}=1$, and $A_{2}=3$, the exact solutions $u_{1}(x, t)$ in Eq. (51) corresponding to the variation of $\alpha, \beta$ are plotted in Fig. 2 as follows. The solution $u_{1}(x, t)$ with $\alpha=\beta=1$ 


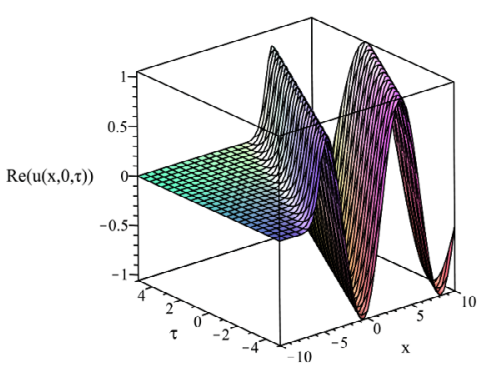

(a) $\alpha=\beta=1$

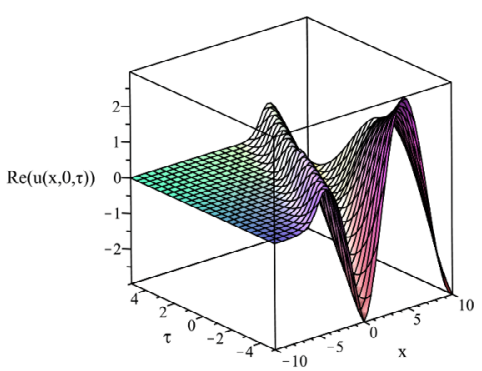

(b) $\alpha=\beta=0.9$

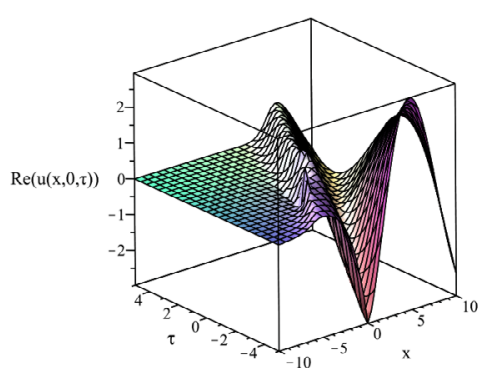

(c) $\alpha=0.9, \beta=0.8$

Figure 1 Associated plots for the real part of $u_{2}(x, z, \tau)$ in Eq. (45) of Eq. (1) on $-10 \leq x \leq 10$ and $-5 \leq \tau \leq 5$ using the modified Kudryashov method

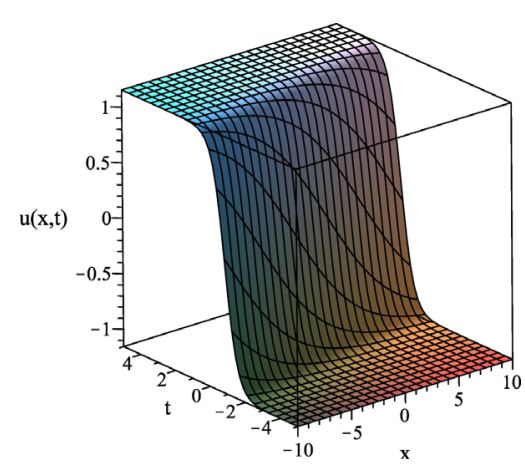

(a) $\alpha=\beta=1$

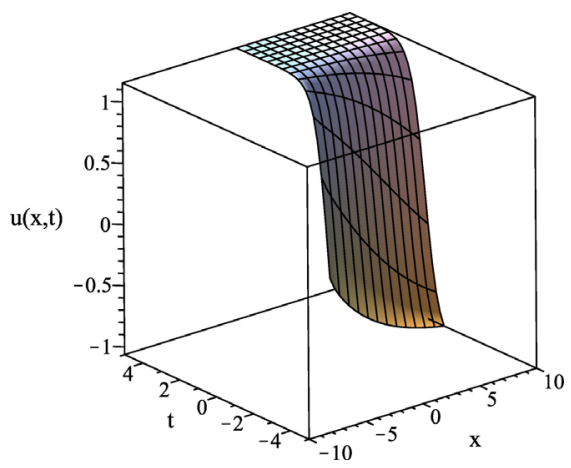

(c) $\alpha=0.9, \beta=0.8$

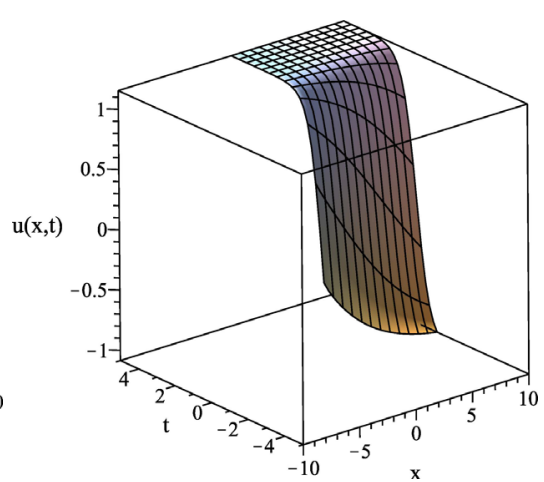

(b) $\alpha=\beta=0.9$

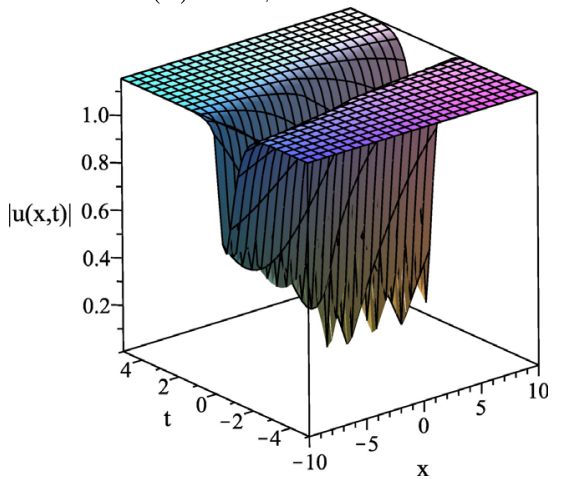

(d) $\alpha=0.9, \beta=0.8$

Figure 2 Associated plots of $u_{1}(x, t)$ in Eq. (51) of Eq. (2) on $-10 \leq x \leq 10$ and $-5 \leq t \leq 5$ using the $\left(G^{\prime} / G, 1 / G\right)$-expansion method 


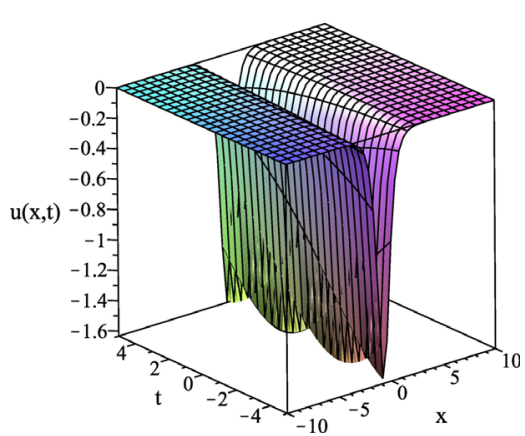

(a) $\alpha=\beta=1$

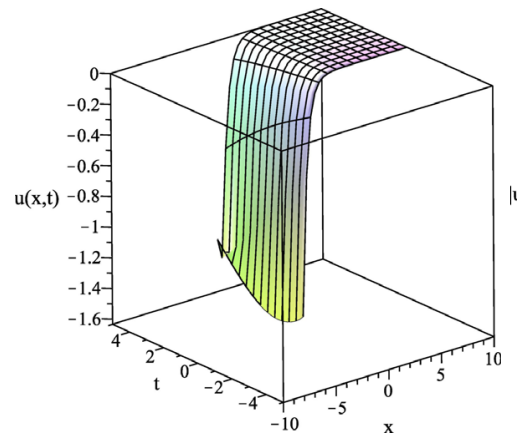

(c) $\alpha=0.9, \beta=0.8$

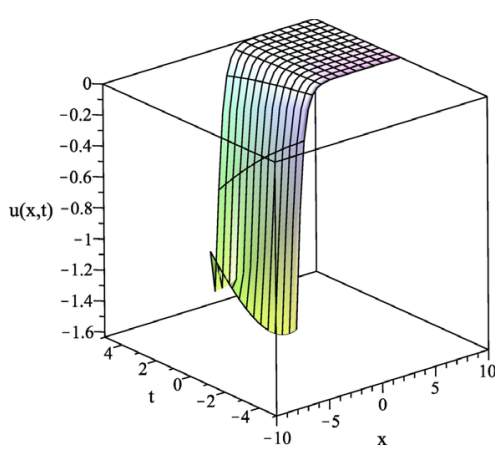

(b) $\alpha=\beta=0.9$

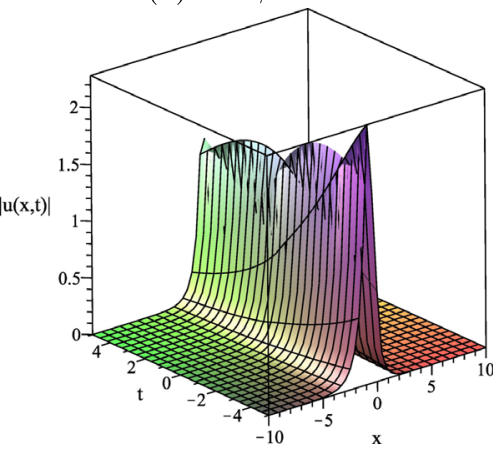

(d) $\alpha=0.9, \beta=0.8$

Figure 3 Associated plots of $u_{2}(x, t)$ in Eq. (53) of Eq. (2) on $-10 \leq x \leq 10$ and $-5 \leq t \leq 5$ using the $\left(G^{\prime} / G, 1 / G\right)$-expansion method

describing the kink-shaped soliton solution is depicted in Fig. 2(a). The graphs of the solution $u_{1}(x, t)$ with $\alpha=\beta=0.9$ and $\alpha=0.9, \beta=0.8$, demonstrating the discontinuous kinkshaped soliton solutions, are presented in Figs. 2(b) and (c), respectively. The graph of $\left|u_{1}(x, t)\right|$ with $\alpha=0.9, \beta=0.8$ is shown in Fig. 2(d).

For the fixed values $m=2, n=-3, k=9, c=1, A_{1}=1$, and $A_{2}=3$, the exact solutions $u_{2}(x, t)$ in Eq. (53) of Eq. (2) corresponding to the variation of $\alpha, \beta \in\{1,0.9,0.8\}$ are scrutinized. The solution $u_{2}(x, t)$ with $\alpha=\beta=1$ describing the anti-soliton solution is plotted in Fig. 3(a). The graphs of the solution $u_{2}(x, t)$ with $\alpha=\beta=0.9$ and $\alpha=0.9, \beta=0.8$ indicating the discontinuous the anti-soliton solution are presented in Figs. 3(b) and (c), respectively. The graph of $\left|u_{2}(x, t)\right|$ with $\alpha=0.9, \beta=0.8$ is plotted in Fig. 3(d).

In Fig. 4(a), we have presented the singular kink-shaped soliton solutions obtained from the solution $u_{3}(x, t)$ in Eq. (55) when the parameter values $m=1, n=-3, k=1, c=9$, $A_{1}=3$, and $A_{2}=1$ and the fractional orders $\alpha=\beta=1$ are used. Using the same parameter values as above but $\alpha=\beta=0.9$ and $\alpha=0.9, \beta=0.8$, the singular single-soliton solution and the singular multiple-soliton solution for $u_{3}(x, t)$ in Eq. (55) are plotted in Figs. 4(b) and (c), respectively. We can see that their graphs are not plotted on the entire domain since these solutions are real only on a certain part of the domain. The graph of $\left|u_{3}(x, t)\right|$ with $\alpha=0.9, \beta=0.8$ is shown in Fig. 4(d).

For the fixed values $m=2, n=-3, k=9, c=1, A_{1}=1$, and $A_{2}=3$, the graphs of the exact solutions $u_{2}(x, t)$ in Eq. (62) of Eq. (2) corresponding to the variation of $\alpha, \beta$ are investigated. The solution $u_{2}(x, t)$ with $\alpha=\beta=1$ describing the singular multiple-soliton solution is depicted in Fig. 5(a). The solutions $u_{2}(x, t)$ with $\alpha=\beta=0.9$ and $\alpha=0.9, \beta=$ 


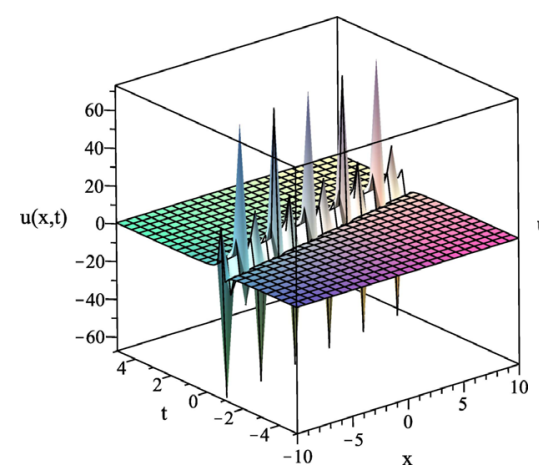

(a) $\alpha=\beta=1$

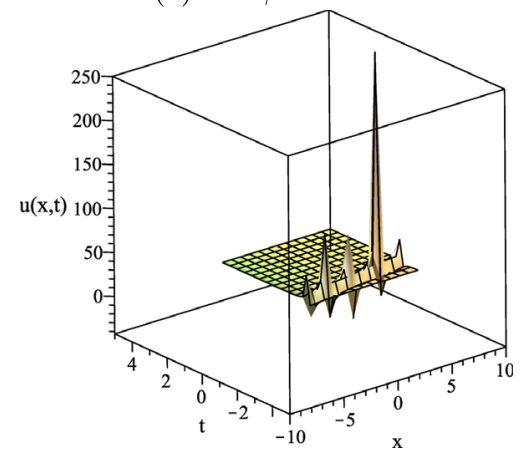

(c) $\alpha=0.9, \beta=0.8$

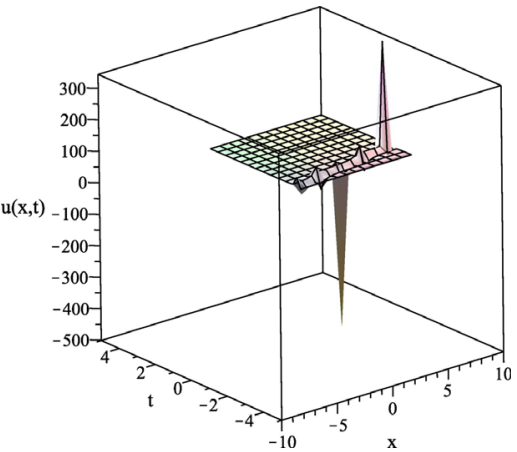

(b) $\alpha=\beta=0.9$

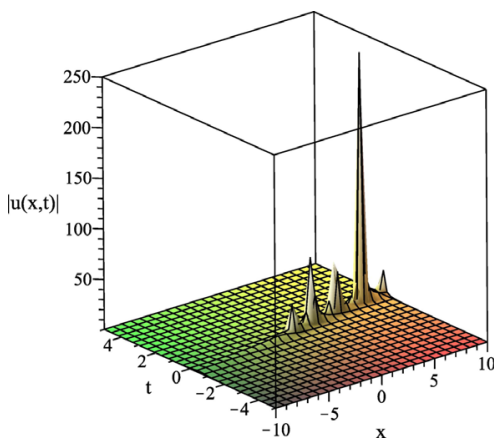

(d) $\alpha=0.9, \beta=0.8$

Figure 4 Associated plots of $u_{3}(x, t)$ in Eq. (55) of Eq. (2) on $-10 \leq x \leq 10$ and $-5 \leq t \leq 5$ using the $\left(G^{\prime} / G, 1 / G\right)$-expansion method

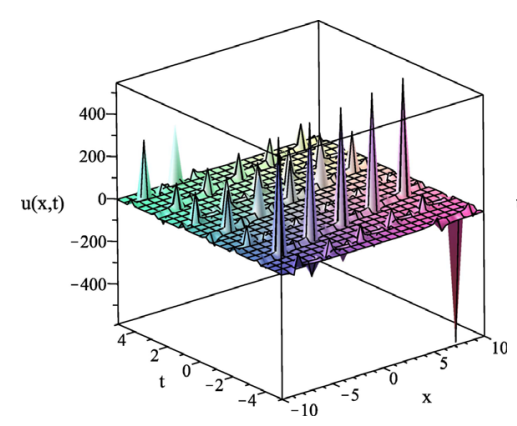

(a) $\alpha=\beta=1$

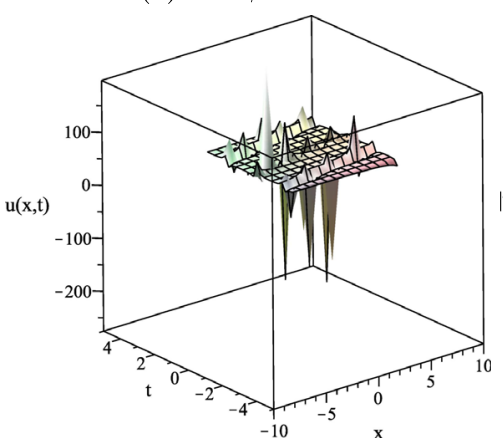

(c) $\alpha=0.9, \beta=0.8$

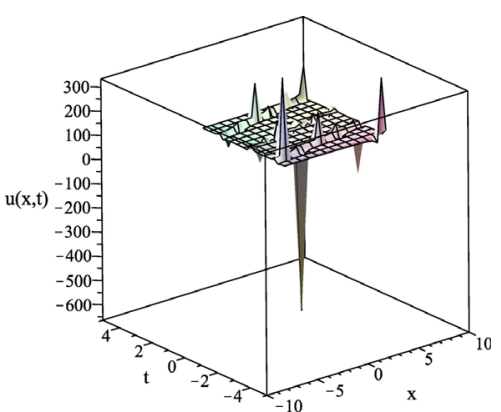

(b) $\alpha=\beta=0.9$

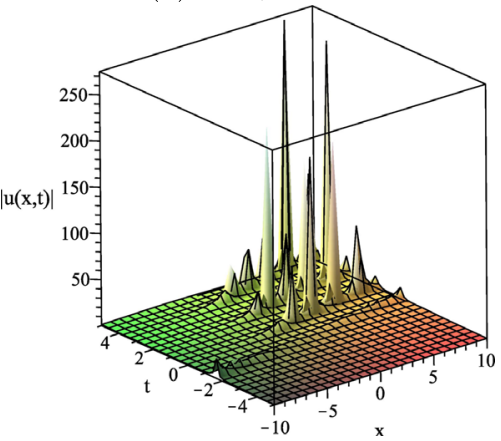

(d) $\alpha=0.9, \beta=0.8$

Figure 5 Associated plots of $u_{2}(x, t)$ in Eq. (62) of Eq. (2) on $-10 \leq x \leq 10$ and $-5 \leq t \leq 5$ using the $\left(G^{\prime} / G, 1 / G\right)$-expansion method 


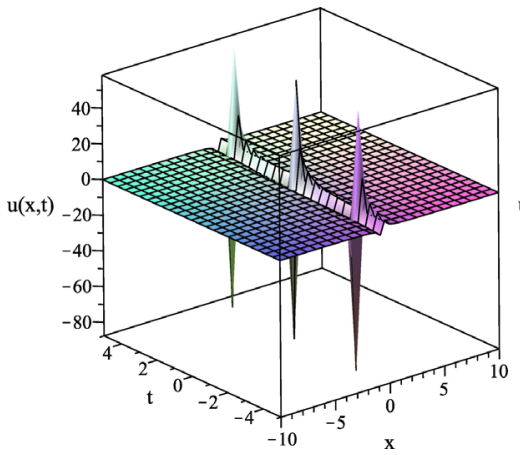

(a) $\alpha=\beta=1$

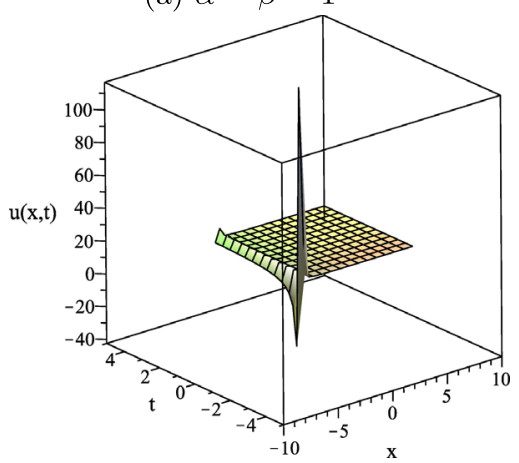

(c) $\alpha=0.9, \beta=0.8$

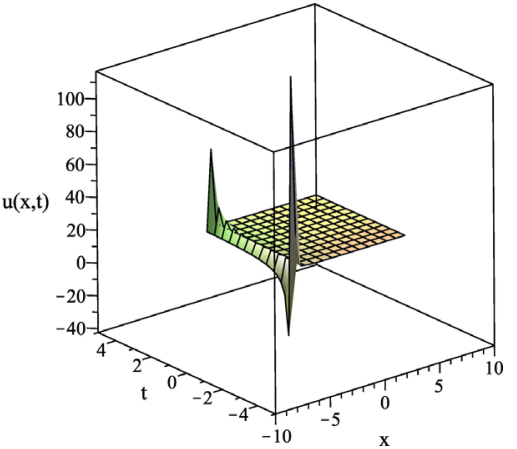

(b) $\alpha=\beta=0.9$

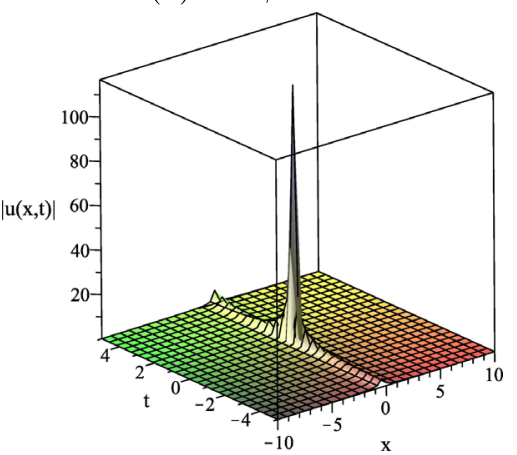

(d) $\alpha=0.9, \beta=0.8$

Figure 6 Associated plots of $u_{1}(x, t)$ in Eq. (69) of Eq. (2) on $-10 \leq x \leq 10$ and $-5 \leq t \leq 5$ using the $\left(G^{\prime} / G, 1 / G\right)$-expansion method

0.8 describing the singular multiple-soliton solution are presented in Figs. 5(b) and (c), respectively. The graph of $\left|u_{2}(x, t)\right|$ with $\alpha=0.9, \beta=0.8$ is plotted in Fig. 5(d).

For the fixed values $m=2, n=-3, k=9, c=1, A_{1}=1$, and $A_{2}=3$, the graphs of the exact solution $u_{1}(x, t)$ in Eq. (69) of Eq. (2) corresponding to the variation of $\alpha, \beta$ are displayed. The solution $u_{2}(x, t)$ with $\alpha=\beta=1$ describing the kink-shaped soliton solutions is depicted in Fig. 6(a). The solutions $u_{1}(x, t)$ with $\alpha=\beta=0.9$ and $\alpha=0.9, \beta=0.8$, showing the singular single-soliton solution, are presented in Figs. 6(b) and (c), respectively. The graph of $\left|u_{1}(x, t)\right|$ with $\alpha=0.9, \beta=0.8$ is shown in Fig. 6(d).

\section{Conclusions}

In this article, we have determined many explicit exact solutions of the nonlinear conformable space-time fractional complex Ginzburg-Landau equation as given by Eq. (1) using the modified Kudryashov method and of the conformable space-time fractional Phi-4 equation as given by Eq. (2) using the $\left(G^{\prime} / G, 1 / G\right)$-expansion method. Some of the obtained solutions are distinct from those found previously via different methods. The fractional complex transform and the symbolic computation package Maple 17 have been used to assist the methods for obtaining the exact solutions of the problems. The exact solutions of Eq. (1) obtained by the modified Kudryashov method are the product of the reciprocal of an exponential function and the exponential function of a purely imaginary function. For Eq. (2), many exact solutions, including hyperbolic function solutions, trigonometric function solutions and rational function solutions, are constructed using the $\left(G^{\prime} / G, 1 / G\right)$ expansion method. Some of these exact solutions have been graphically characterized in 
terms of a variety of distinct physical structures such as an oscillatory wave solution, a kink-shaped soliton solution, a singular single-soliton solution, a singular multiple-soliton solution and an anti-soliton solution. Some of our obtained exact solutions are similar to the solutions in [36] which was solved using the $\left(G^{\prime} / G\right)$-expansion method. However, our solutions are the generalization of their solutions because the $\left(G^{\prime} / G, 1 / G\right)$-expansion method can be reduced to the $\left(G^{\prime} / G\right)$-expansion method by setting the parameter $\mu$ in Eq. (11) and the coefficient $b_{j}$ in Eq. (20) to be zero. Therefore, the $\left(G^{\prime} / G, 1 / G\right)$-expansion method is an extension of the $\left(G^{\prime} / G\right)$-expansion method. The applications of these solutions have been found in many physical phenomena such as optical fibers and nuclear physics. All of our results obtained by the two methods have been attained and verified by substituting them back into the original problems with the aid of the Maple 17 package program. Since the two methods are powerful, systematic and reliable for obtaining the exact solutions of the equations, they could be applied efficiently for a great many NPDEs including fractional-order ones arising in mathematical physics.

\section{Acknowledgements}

The authors would like to express their sincere thanks to the anonymous referees for their time and valuable comments.

\section{Funding}

This research was funded by King Mongkut's University of Technology North Bangkok under contract no. KMUTNB-61-KNOW-037.

\section{Competing interests}

The authors declare that they have no competing interests.

Authors' contributions

All authors worked together to produce the results, read and approved the final manuscript.

\section{Publisher's Note}

Springer Nature remains neutral with regard to jurisdictional claims in published maps and institutional affiliations.

Received: 1 February 2019 Accepted: 22 May 2019 Published online: 31 May 2019

\section{References}

1. Guo, F., Lin, J.: Interaction solutions between lump and stripe soliton to the $(2+1)$-dimensional Date-Jimbo-Kashiwara-Miwa equation. Nonlinear Dyn. 96(2), 1233-1241 (2019)

2. Wazwaz, A.-M., Kaur, L.: Optical solitons and Peregrine solitons for nonlinear Schrödinger equation by variational iteration method. Optik 179, 804-809 (2019)

3. Naghshband, S., Araghi, M.A.F.: Solving generalized quintic complex Ginzburg-Landau equation by homotopy analysis method. Ain Shams Eng. J. 9(4),607-613 (2018)

4. Seadawy, A.R.: Solitary wave solutions of two-dimensional nonlinear Kadomtsev-Petviashvili dynamic equation in dust-acoustic plasmas. Pramana 89(3), 1-11 (2017)

5. Seadawy, A.R.: Three-dimensional nonlinear modified Zakharov-Kuznetsov equation of ion-acoustic waves in a magnetized plasma. Comput. Math. Appl. 71(1), 201-212 (2016)

6. Mahmood, B.A., Yousif, M.A.: A novel analytical solution for the modified Kawahara equation using the residual power series method. Nonlinear Dyn. 89(2), 1233-1238 (2017)

7. Seadawy, A.R.: The generalized nonlinear higher order of KdV equations from the higher order nonlinear Schrödinger equation and its solutions. Optik 139, 31-43 (2017)

8. Ayub, K., Khan, M.Y., Mahmood-UI-Hassan, Q.: Solitary and periodic wave solutions of Calogero-Bogoyavlenskii-Schiff equation via exp-function methods. Comput. Math. Appl. 74, 3231-3241 (2017)

9. Qin, Z., Mu, G., Ma, H.: (G'/G)-Expansion method for the fifth-order forms of KdV-Sawada-Kotera equation. Appl. Math. Comput. 222, 29-33 (2013)

10. Taghizadeh, N., Mirzazadeh, M.: The first integral method to some complex nonlinear partial differential equations. J. Comput. Appl. Math. 235, 4871-4877 (2011)

11. Helal, M., Seadawy, A.R.: Benjamin-Feir instability in nonlinear dispersive waves. Comput. Math. Appl. 64(11), 3557-3568 (2012)

12. Podlubny, I.: Fractional Differential Equations: An Introduction to Fractional Derivatives, Fractional Differential Equations, to Methods of Their Solution and Some of Their Applications. Academic Press, San Diego (1999)

13. Caputo, M., Fabrizio, M.: A new definition of fractional derivative without singular kernel. Prog. Fract. Differ. Appl. 1(2)، $1-13(2015)$

14. Li, C., Guo, Q., Zhao, M.: On the solutions of $(2+1)$-dimensional time-fractional Schrödinger equation. Appl. Math. Lett. 94, 238-243 (2019) 
15. Duan, J.-S., Chaolu, T., Rach, R.: Solutions of the initial value problem for nonlinear fractional ordinary differential equations by the Rach-Adomian-Meyers modified decomposition method. Appl. Math. Comput. 218, 8370-8392 (2012)

16. Wu, G.-C., Baleanu, D.: Variational iteration method for the Burgers' flow with fractional derivatives - new Lagrange multipliers. Appl. Math. Model. 37, 6183-6190 (2013)

17. Jiang, Y., Ma, J.: High-order finite element methods for time-fractional partial differential equations. J. Comput. Appl. Math. 235, 3285-3290 (2011)

18. Zayernouri, M., Matzavinos, A.: Fractional Adams-Bashforth/Moulton methods: an application to the fractional Keller-Segel chemotaxis system. J. Comput. Phys. 317, 1-14 (2016)

19. Shakeel, M., Mohyud-Din, S.T.: Soliton solutions for the positive Gardner-KP equation by $\left(G^{\prime} / G, 1 / G\right)$-expansion method. Ain Shams Eng. J. 5, 951-958 (2014)

20. Demiray, S., Ömer, Ü., Bekir, A.: Exact solutions of nonlinear wave equations using $\left(G^{\prime} / G, 1 / G\right)$-expansion method. J. Egypt. Math. Soc. 23, 78-84 (2015)

21. Zayed, E.M.E., Alurrfi, K.A.E.: The (G'/G,1/G)-expansion method and its applications to two nonlinear Schrödinger equations describing the propagation of femtosecond pulses in nonlinear optical fibers. Optik 127, 1581-1589 (2016)

22. Sirisubtawee, S., Koonprasert, S., Khaopant, C., Porka, W.: Two reliable methods for solving the $(3+1)$-dimensional space-time fractional Jimbo-Miwa equation. Math. Probl. Eng. 2017, 30 (2017)

23. Ege, S.M., Misirli, E.: The modified Kudryashov method for solving some fractional-order nonlinear equations. Adv Differ. Equ. 2014, 135 (2014)

24. Zayed, E.M.E., Alurrfi, K.: The modified Kudryashov method for solving some seventh order nonlinear PDEs in mathematical physics. World J. Model. Simul. 11, 308-319 (2015)

25. Hosseini, K., Mayeli, P., Ansari, R.: Modified Kudryashov method for solving the conformable time-fractional Klein-Gordon equations with quadratic and cubic nonlinearities. Optik 130, 737-742 (2017)

26. Kumar, D., Seadawy, A.R., Joardar, A.K.: Modified Kudryashov method via new exact solutions for some conformable fractional differential equations arising in mathematical biology. Chin. J. Phys. 56, 75-85 (2018)

27. Gómez, S.C.A.: A nonlinear fractional Sharma-Tasso-Olver equation: new exact solutions. Appl. Math. Comput. 266, 385-389 (2015)

28. Ilie, M., Biazar, J., Ayati, Z.: The first integral method for solving some conformable fractional differential equations. Opt. Quantum Electron. 50(2), 55 (2018)

29. Ilie, M., Biazar, J., Ayati, Z.: Analytical study of exact traveling wave solutions for time-fractional nonlinear Schrödinger equations. Opt. Quantum Electron. 50(12), 413 (2018)

30. Ilie, M., Biazar, J., Ayati, Z.: Resonant solitons to the nonlinear Schrödinger equation with different forms of nonlinearities. Optik 164, 201-209 (2018)

31. Shi, Y., Dai, Z., Li, D.: Application of Exp-function method for 2D cubic-quintic Ginzburg-Landau equation. Appl. Math. Comput. 210, 269-275 (2009)

32. Zayed, E.M.E., Alurrfi, K.A.E.: New extended auxiliary equation method and its applications to nonlinear Schrödinger-type equations. Optik 127, 9131-9151 (2016)

33. Zayed, E.M.E., Al-Nowehy, A.-G.: Solitons and other exact solutions for a class of nonlinear Schrödinger-type equations. Optik 130, 1295-1311 (2017)

34. Akter, J., Akbar, M.A.: Exact solutions to the Benney-Luke equation and the Phi-4 equations by using modified simple equation method. Results Phys. 5, 125-130 (2015)

35. Tariq, H., Akram, G.: New approach for exact solutions of time fractional Cahn-Allen equation and time fractional Phi-4 equation. Phys. A, Stat. Mech. Appl. 473, 352-362 (2017)

36. Khan, U., Ellahi, R., Ullah, R., Mohyud-Din, S.T.: Extracting new solitary wave solutions of Benny-Luke equation and Phi-4 equation of fractional order by using $\left(G^{\prime} / G\right)$-expansion method. Opt. Quantum Electron. 50, 379-389 (2018)

37. Akram, G., Batool, F., Riaz, A.: Two reliable techniques for the analytical study of conformable time-fractional Phi-4 equation. Opt. Quantum Electron. 50, 22 (2017)

38. Khalil, R., Horani, M.A., Yousef, A., Sababheh, M.: A new definition of fractional derivative. J. Comput. Appl. Math. 264 $65-70(2014)$

39. Ryabov, P., Sinelshchikov, D., Kochanov, M.: Application of the Kudryashov method for finding exact solutions of the high order nonlinear evolution equations. Appl. Math. Comput. 218, 3965-3972 (2011)

40. Hubert, M.B., Betchewe, G., Doka, S.Y., Crepin, K.T.: Soliton wave solutions for the nonlinear transmission line using the Kudryashov method and the (G'/G)-expansion method. Appl. Math. Comput. 239, 299-309 (2014)

41. Raslan, K.R., EL-Danaf, T.S., Ali, K.K.: Exact solution of the space-time fractional coupled EW and coupled MEW equations. Eur. Phys. J. Plus 132, 319 (2017)

42. Li, B., Chen, Y., Zhang, H.: Explicit exact solutions for new general two-dimensional KdV-type and two-dimensional KdV-Burgers-type equations with nonlinear terms of any order. J. Phys. A, Math. Gen. 35, 8253 (2002) 